Cite this: Phys. Chem. Chem. Phys., 2014, 16, 3466

Received 4th November 2013, Accepted 23rd December 2013

DOI: $10.1039 / \mathrm{c} 3 \mathrm{cp} 54664 \mathrm{k}$

www.rsc.org/pccp

\section{A combined experimental and theoretical study of reactions between the hydroxyl radical and oxygenated hydrocarbons relevant to astrochemical environments}

\author{
R. J. Shannon, ${ }^{a}$ R. L. Caravan, ${ }^{a}$ M. A. Blitz ${ }^{a b}$ and D. E. Heard ${ }^{\star a b}$
}

\begin{abstract}
The kinetics of the reactions of the hydroxyl radical $(\mathrm{OH})$ with acetone and dimethyl ether (DME) have been studied between 63-148 $\mathrm{K}$ and at a range of pressures using laser-flash photolysis coupled with laser induced fluorescence detection of $\mathrm{OH}$ in a pulsed Laval nozzle apparatus. For acetone, a large negative temperature dependence was observed, with the rate coefficient increasing from $k_{1}=$ $(1.6 \pm 0.8) \times 10^{-12} \mathrm{~cm}^{3}$ molecule ${ }^{-1} \mathrm{~s}^{-1}$ at $148 \mathrm{~K}$ to $(1.0 \pm 0.1) \times 10^{-10} \mathrm{~cm}^{3}$ molecule ${ }^{-1} \mathrm{~s}^{-1}$ at $79 \mathrm{~K}$, and also increasing with pressure. For DME, a similar behaviour was found, with the rate coefficient increasing from $k_{2}=(3.1 \pm 0.5) \times 10^{-12} \mathrm{~cm}^{3}$ molecule ${ }^{-1} \mathrm{~s}^{-1}$ at $138 \mathrm{~K}$ to $(1.7 \pm 0.1) \times 10^{-11} \mathrm{~cm}^{3}$ molecule $\mathrm{s}^{-1}$ at $63 \mathrm{~K}$, and also increasing with pressure. The temperature and pressure dependence of the experimental rate coefficients are rationalised for both reactions by the formation and subsequent stabilisation of a hydrogen bonded complex, with a non-zero rate coefficient extrapolated to zero pressure supportive of quantum mechanical tunnelling on the timescale of the experiments leading to products. In the case of $\mathrm{DME}$, experiments performed in the presence of $\mathrm{O}_{2}$ provide additional evidence that the yield of the $\mathrm{CH}_{3} \mathrm{OCH}_{2}$ abstraction product, which can recycle $\mathrm{OH}$ in the presence of $\mathrm{O}_{2}$, is $\geq 50 \%$. The experimental data are modelled using the MESMER (Master Equation Solver for Multi Energy Well Reactions) code which includes a treatment of quantum mechanical tunnelling, and uses energies and structures of transition states and complexes calculated by ab initio methods. Good agreement is seen between experiment and theory, with MESMER being able to reproduce for both reactions the temperature behaviour between $\sim 70-800 \mathrm{~K}$ and the pressure dependence observed at $\sim 80 \mathrm{~K}$. At the limit of zero pressure, the model predicts a rate coefficient of $\sim 10^{-11} \mathrm{~cm}^{3}$ molecule ${ }^{-1} \mathrm{~s}^{-1}$ for the reaction of $\mathrm{OH}$ with acetone at $20 \mathrm{~K}$, providing evidence that the reaction can proceed quickly in those regions of space where both species have been observed. The results and modelling build considerably on our previous experimental study performed under a much more limited range of conditions (Shannon et al., Phys. Chem. Chem. Phys., 2010, 12, 13511-13514).
\end{abstract}

\section{Introduction}

Rate coefficients are experimentally challenging to measure below $\sim 200 \mathrm{~K}$ owing to the condensation of at least one of the reagents at the walls of the reaction chamber, and Laval nozzle techniques have enjoyed considerable success for kinetic studies in this temperature regime. ${ }^{2-9}$ It is firmly established that many neutral-neutral reactions are fast at very low temperatures with some exhibiting rate coefficients close to the gas kinetic limit. The majority of experimental studies have involved barrierless reactions which possess an attractive potential energy surface for

\footnotetext{
${ }^{a}$ School of Chemistry, University of Leeds, Leeds, LS2 9JT, UK. E-mail: d.e.Heard@leeds.ac.uk

${ }^{b}$ National Centre for Atmospheric Science, University of Leeds, Leeds, LS2 9JT, UK
}

the approach of the reagents. ${ }^{10}$ The rate coefficients for such reactions are also normally significant at room temperature ${ }^{11}$ and typically display a weak increase in the rate coefficient as the temperature is lowered. However, recent work by Shannon et al. have shown that another class of reaction with an overall barrier to reaction can also be fast at low temperatures. ${ }^{1,5}$ This class of reaction involves hydrogen atom abstraction from small oxygenated volatile organic molecules (OVOCs) by the $\mathrm{OH}$ radical, with the formation of a hydrogen-bonded complex prior to abstraction facilitating a large increase in the reaction rate coefficients at low temperatures. In a previous publication, ${ }^{1}$ we reported the low temperature kinetic behaviour of two such systems, namely the reactions of $\mathrm{OH}$ with acetone and dimethyl ether (DME), but under a very limited range of low temperatures, and at a single pressure. 
Both of these reactions are important in combustion systems and in the Earth's atmosphere, with DME having been proposed as a potential diesel substitute. ${ }^{12}$ In addition, as shown in our earlier work for these reactions and for methyl ethyl ketone ${ }^{1}$ and also for methanol, ${ }^{5}$ reactions between the $\mathrm{OH}$ radical and OVOCs are potentially important in very low temperature extraterrestrial environments such as star-forming regions and the interstellar medium. $\mathrm{OH}$ and several OVOCs have been observed by astronomers in cold, dense molecular clouds ${ }^{13}$ In particular $\mathrm{OH}$, acetone and DME have all been observed in the Sagittarius B2 molecular cloud. ${ }^{14-16}$ Unfortunately, very few rate coefficients for reactions of $\mathrm{OH}$ with OVOCs have been measured below $200 \mathrm{~K}$, and consequently the kinetic databases used for astrochemical modelling contain very little information about this type of reaction. ${ }^{17}$

Owing to their importance in both atmospheric and combustions systems, rate coefficients for the hydrogen-atom abstraction reactions of $\mathrm{OH}$ with both acetone (R1) and DME (R2) have been measured by a large number of groups at temperatures above $200 \mathrm{~K}$,

$$
\begin{array}{r}
\mathrm{OH}+\mathrm{CH}_{3} \mathrm{C}(\mathrm{O}) \mathrm{CH}_{3} \stackrel{k_{1}}{\rightarrow} \mathrm{CH}_{3} \mathrm{C}(\mathrm{O}) \mathrm{CH}_{2}+\mathrm{H}_{2} \mathrm{O} \\
\mathrm{OH}+\mathrm{CH}_{3} \mathrm{OCH}_{3} \stackrel{k_{2}}{\rightarrow} \mathrm{CH}_{3} \mathrm{OCH}_{2}+\mathrm{H}_{2} \mathrm{O}
\end{array}
$$

where $k_{1}$ and $k_{2}$ are the overall rate coefficients. Both reactions can be described by the same generic type of potential energy surface involving a looser, outer (TS1) and a tighter, inner (TS2) transition state as suggested by Greenwald et al., ${ }^{18,19}$ a simplified schematic of which is shown in Fig. 1. The potential includes the formation of a hydrogen-bonded complex prior to the activation barrier to hydrogen atom abstraction. At high temperatures the kinetic behaviour of both reactions is dominated by TS 2 which coincides

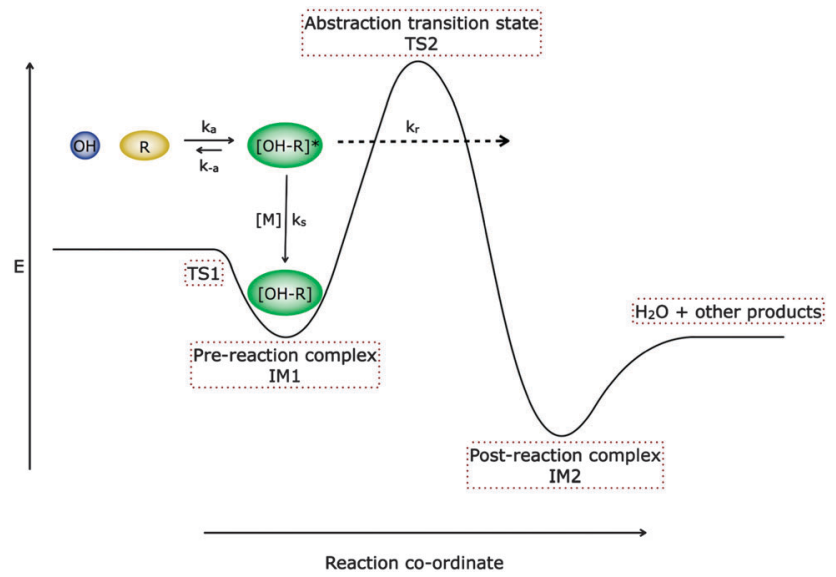

Fig. 1 Schematic potential energy surface demonstrating the two transition state model of Greenwald et al. ${ }^{18,19}$ The potential energy surface represents the formation of a hydrogen bonded complex (IM1) with an associated microscopic rate coefficient, $k_{\mathrm{a}}$. The complex can either be stabilised (with a corresponding microscopic rate coefficient of $k_{\mathrm{s}}[M]$ where $[M]$ is the total gas density), re-dissociate back to reactants (with microscopic rate coefficient $k_{-a}$ ), or form abstraction products via a post-reaction complex IM2 (with microscopic rate coefficient $k_{\mathrm{r}}$ ), either by surmounting or tunnelling through the barrier to reaction (TS2).

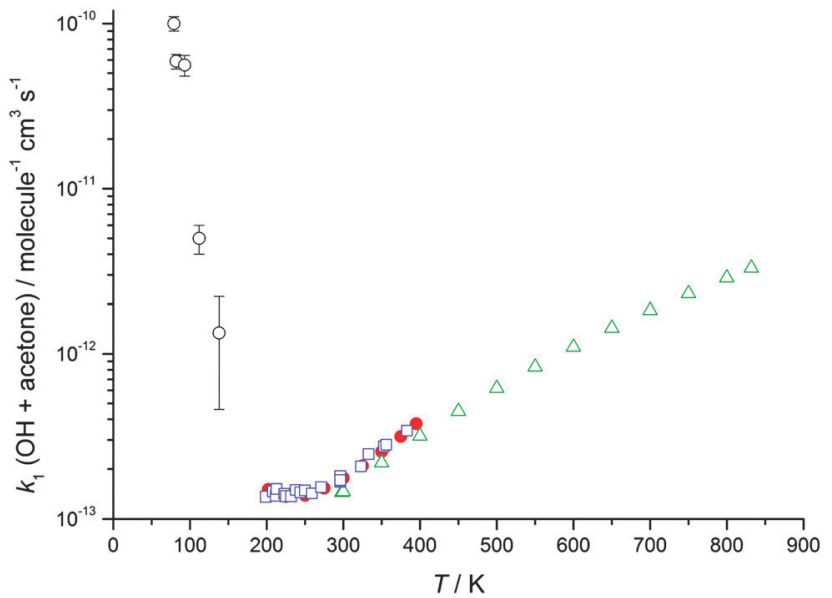

Fig. 2 The temperature dependence of the experimentally determined rate coefficients for the reaction of $\mathrm{OH}$ with acetone. Open green triangles: Yamada et al. ${ }^{39}$ Filled red circles: Wollenhaupt et al. ${ }^{52}$ Open blue squares: Gierczak et al. ${ }^{53}$ Open black circles: this work (total density in $\mathrm{N}_{2}$ ranging from (5.1-17) $\times 10^{16}$ molecule $\mathrm{cm}^{-3}$ ). Error bars as for Table 1 .

with the energy barrier to products. As a consequence of this, the rate coefficients increase with temperature as shown in Fig. 2 and 3 for acetone and DME, respectively. However, at temperatures lower than $\sim 300 \mathrm{~K}$, there is deviation from Arrhenius behaviour, as manifested by the rate coefficients for both reactions becoming more temperature independent, and indeed, in the case of acetone, a slight negative temperature dependence is observed close to $200 \mathrm{~K}$. These results provide evidence for a change in mechanism from a simple abstraction over the barrier to reaction. A previous study in this laboratory using the pulsed Laval nozzle apparatus represents the only measurement of the rate coefficients for (R1) and (R2) below $195 \mathrm{~K}$, and for both reactions a significant enhancement in the rate coefficient was observed. ${ }^{1}$ For the reaction of $\mathrm{OH}$ with acetone $k_{1}$ was observed to

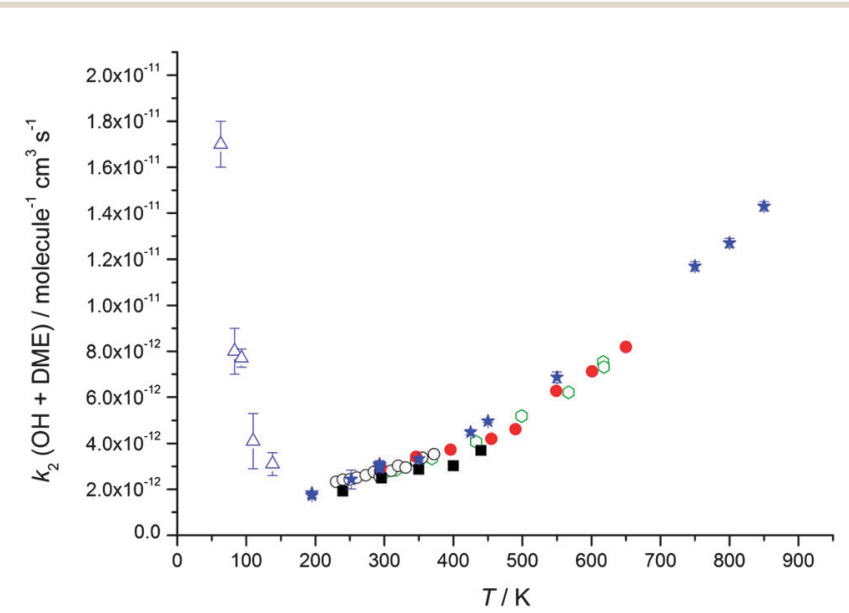

Fig. 3 Temperature dependence of the experimentally determined rate coefficients for the reaction of $\mathrm{OH}$ with dimethyl ether (DME). Green open hexagons: Bonard et al. ${ }^{49}$ Red filled circles: Arif et al. ${ }^{48}$ Black open circles: Mellouki et al. ${ }^{47}$ Black filled squares: Wallington et al. ${ }^{54}$ Blue filled stars: Carr et al. ${ }^{32}$ Blue open triangles: this work (total density in $\mathrm{N}_{2}$ ranging from $(3.4-9.1) \times 10^{16}$ molecule $\mathrm{cm}^{-3}$ ). Error bars as for Table 2 . 
increase by a factor of 334 between $295 \mathrm{~K}$ and $86 \mathrm{~K}^{1}$ At very low temperatures there is insufficient collision energy to access TS2 and so the transition state is located at TS1, and the rate coefficient will be limited by long range forces to form the hydrogen-bonded complex. For the same reaction, Caralp et $a .^{20}$ provided evidence through calculations that the deviation from Arrhenius behaviour below $250 \mathrm{~K}$ could be attributed to quantum mechanical tunnelling to abstraction products. However, Wallington and Kurylo ${ }^{21}$ postulated that stabilisation into the pre-reaction complex (IM1) is a possible reaction pathway at lower temperatures. Analogous reaction pathways are likely to operate in the reaction of $\mathrm{OH}$ with DME, but regardless of whether at very low temperatures the mechanism is dominated by tunnelling to products and/or collisional stabilisation, the weakly bound pre-reaction complex (IM1) is likely to be important. These weakly bound hydrogen-bonded complexes are ubiquitous in reactions between the $\mathrm{OH}$ radical and OVOCs, ${ }^{5,22}$ with typical binding energies of a few $10 \mathrm{~s}$ of $\mathrm{kJ} \mathrm{mol}^{-1}$, but their role in controlling the temperature dependence of the rate coefficients of these reactions is still poorly understood.

In this paper, we report rate coefficients for the reactions of $\mathrm{OH}$ with acetone and DME determined over a much wider range of conditions compared with our previous study ${ }^{1}$ in order to further probe the role of the pre-reaction complex as well as quantum mechanical tunnelling. ${ }^{23}$ The extent of deviation from Arrhenius behaviour as well as the contribution of the two mechanisms suggested by Caralp et $a .^{20}$ and Wallington and Kurylo $^{21}$ to the overall rate coefficient are investigated through a series of kinetic measurements as a function of temperature and pressure using a pulsed Laval nozzle apparatus, and modelling using the MESMER (Master Equation Solver for Multi Energy Well Reactions) package. ${ }^{24}$

\section{Experimental procedure}

The Leeds pulsed Laval nozzle apparatus has been described in detail previously, ${ }^{5,25}$ and only a brief description will be given here. The reaction mixture consisting of the radical precursor, the reagent and the bath gas $\left(\mathrm{N}_{2}\right)$ is admitted into a $1 \mathrm{~cm}^{3}$ stainless steel reservoir via two pulsed valves (Parker series 9) fired at $5 \mathrm{~Hz}$ repetition frequency with a pulse duration of $\sim 10 \mathrm{~ms}$. This gas mixture is then expanded through a convergentdivergent Laval nozzle into a stainless steel reaction chamber held at low pressure. The reaction chamber is pumped by a Leybold RUVAC 251 roots pump backed by a Leybold D65B rotary pump and the pressure is recorded by two capacitance manometers (Leybold CTR 90 0-1000 Torr and 0-10 Torr). The pumping system is throttled with a specially adapted gate valve to allow control of the chamber pressure to \pm 0.01 Torr.

Various mixtures of nitrogen (BOC, OFN, >99.998\%), acetone (VWR, >99\%), DME (Aldrich >99.9\%), acetone-d $\mathrm{d}_{6}$ (Eur-isotop, $>99.8 \%), t$-butyl-hydroperoxide $\left(\left(\mathrm{CH}_{3}\right)_{3} \mathrm{OOH}=t\right.$-BuOOH, Aldrich, $70 \% \mathrm{wt}$ aqueous solution) or oxygen (BOC, analytical grade) were flowed in to a $1 \mathrm{~L}$ stainless steel ballast tank via a set of calibrated mass flow controllers (MKS instruments) to enable thorough mixing.
For experiments where $t$-BuOOH was used as the $\mathrm{OH}$ precursor, nitrogen was passed through a bubbler containing liquid $t$ - $\mathrm{BuOOH}$ which was then entrained in the gas flow. Acetone, DME or acetone- $\mathrm{d}_{6}$ were purified using freeze-pump-thaw cycles on a vacuum line. The vapour pressures of acetone, DME or acetone- $\mathrm{d}_{6}$ were then admitted to an empty cylinder and were diluted with nitrogen and left overnight prior to use to ensure full mixing.

The temperature, density and pressure within the Laval nozzle expansion are all related to their stagnation, pre-expansion values by the Mach number, $M$. The stagnation pressure $P_{\mathrm{o}}$ in the preexpansion region of the reservoir and the impact pressure $P_{\mathrm{i}}$ were both measured, and are related to $M$ by: ${ }^{25}$

$$
\frac{P_{\mathrm{i}}}{P_{\mathrm{o}}}=\left[\frac{(\gamma+1) M^{2}}{(\gamma-1) M^{2}+2}\right]^{\frac{\gamma}{\gamma-1}}\left(\frac{\gamma+1}{2 \gamma M^{2}-\gamma+1}\right)^{\frac{1}{\gamma-1}}
$$

where $\gamma=C_{\mathrm{p}} / C_{\mathrm{v}}$ is the ratio of specific heat capacities, which for the carrier $\mathrm{N}_{2}$ gas used in this work is $7 / 5$. A trial value of $M$ is chosen and its value optimised iteratively in order to find the value which best matches the measured value of $P_{\mathrm{i}} / P_{\mathrm{o}}$. Having determined the Mach number, the temperature in the expansion is obtained using:

$$
\frac{T_{0}}{T}=1+\frac{\gamma-1}{2} M^{2}
$$

where $T_{0}$ is the measured temperature in the stagnation region. The total gas density in the supersonic expansion, $\rho$, is then given by:

$$
\frac{\rho_{0}}{\rho}=\left(\frac{T_{0}}{T}\right)^{1 /(\gamma-1)}
$$

where $\rho_{0}$ is the gas density in the pre-expansion reservoir. Several Laval nozzles have been designed to give temperatures in the supersonic jet expansion in the range 63-148 K. By varying the flow backing pressure in the pre-expansion reservoir through changing the gas flow rate, a number of stable flows of different gas densities have been found using the same nozzle. The temperature profile within the supersonic expansions used in this study have been characterised using the impact pressure measurement technique and rotationally-resolved laser-induced fluorescence spectroscopy (LIF), as described previously. ${ }^{25}$

$\mathrm{OH}$ radicals were generated using two methods. For the reaction of $\mathrm{OH}$ with acetone, the $\mathrm{OH}$ radicals were usually produced via $248 \mathrm{~nm}$ photolysis of acetone itself using a $\mathrm{KrF}$ excimer laser (Lambda Physik LPX200, $5 \mathrm{~Hz}$ ) in the presence of oxygen:

$$
\begin{gathered}
\mathrm{CH}_{3} \mathrm{C}(\mathrm{O}) \mathrm{CH}_{3}+h \nu \rightarrow \mathrm{CH}_{3} \mathrm{CO}+\mathrm{CH}_{3} \\
\mathrm{CH}_{3} \mathrm{CO}+\mathrm{O}_{2} \stackrel{k_{4}}{\rightarrow} \mathrm{OH}+\text { co-product }
\end{gathered}
$$

where $k_{4}$ is the rate coefficient for reaction (R4). This method, as fully discussed by Carr et al., ${ }^{26}$ eliminates the need for additional reagents other than $\mathrm{O}_{2}$ to be introduced into the chamber. For the experiments of $\mathrm{OH}$ with acetone using a nozzle designed for $\sim 140 \mathrm{~K}$, and also for all experiments studying the reaction of $\mathrm{OH}$ with $\mathrm{DME}$, the $248 \mathrm{~nm}$ photolysis of $t$ - $\mathrm{BuOOH}$ was used as an $\mathrm{OH}$ precursor:

$$
\left(\mathrm{CH}_{3}\right)_{3} \mathrm{COOH}+h \nu \rightarrow\left(\mathrm{CH}_{3}\right)_{3} \mathrm{CO}+\mathrm{OH}
$$


In all experiments the $\mathrm{OH}$ radicals were then probed by LIF at $\sim 282 \mathrm{~nm}$ via the $\mathrm{A}^{2} \Sigma^{+} \leftarrow \mathrm{X}^{2} \Pi_{\mathrm{i}}(1,0) \mathrm{Q}_{1}(1.5)$ transition using a $532 \mathrm{~nm}$ frequency-doubled Nd:YAG laser (Litron LPY 664) pumped dye laser (Sirah Gmbh-Cobra Stretch, Rhodamine 6G dye in methanol) system. The photolysis and probe lasers were at right angles to one another with the excimer pump laser directed along the axis of the pulsed expansion. The offresonant fluorescence was collected on a third axis mutually perpendicular to the two laser beams using a photomultiplier (Thorn EMI 9813) fitted with a $308 \mathrm{~nm}$ interference filter (Barr Associates, $308.5 \pm 5.5 \mathrm{~nm}$ ) and the signal was digitised and integrated using a digital oscilloscope (LeCroy Waverunner-2). For experiments in which OD was monitored following its formation in the reaction of $\mathrm{CD}_{3} \mathrm{CO}+\mathrm{O}_{2}$, an analogous transition to $\mathrm{OH}$ was used, with a laser excitation and fluorescence collection wavelengths of 287 and $308 \mathrm{~nm}$, respectively. By varying the time delay between the photolysis and probe lasers using a delay generator (Berkeley Nucleonics Corporation model 555), the temporal evolution of the relative $\mathrm{OH}$ concentration was recorded. The fluorescence signal was normalised for fluctuations in the dye laser power which was measured simultaneously using a photodiode. All experiments were conducted under pseudo-first-order conditions ([reagent] $\gg[\mathrm{OH}]$ ) and the reagent concentration was varied from $\sim 0.1$ to $1 \%$ of the total gas flow.

When the reaction of the acetyl radical with $\mathrm{O}_{2}(\mathrm{R} 4)$ was used as the source of $\mathrm{OH}$, the temporal profile of the $\mathrm{OH}$ concentration, which is proportional to the fluorescence intensity, is given by:

$$
[\mathrm{OH}]_{t}=\frac{A\left[\mathrm{CH}_{3} \mathrm{CO}\right]_{0} k_{4}^{\prime}}{k_{4}^{\prime}-\left(k_{1}^{\prime}+k_{\mathrm{diff}}\right)}\left[\exp \left(-\left(k_{1}^{\prime}+k_{\text {diff }}\right) t\right)-\exp \left(-k_{4}^{\prime} t\right)\right]
$$

where $\left[\mathrm{CH}_{3} \mathrm{CO}\right]_{0}$ is the initial concentration of $\mathrm{CH}_{3} \mathrm{CO}$ from (R3), $k_{4}{ }^{\prime}=k_{4}\left[\mathrm{O}_{2}\right]$ and $k_{1}{ }^{\prime}=k_{1}\left[\mathrm{CH}_{3} \mathrm{C}(\mathrm{O}) \mathrm{CH}_{3}\right]$ are the pseudo first order rate coefficients for reactions (R1) and (R4), respectively, $t$ is the reaction time and $A$ is a scaling factor reflecting the non-unity yield of $\mathrm{OH}$ from (R4). ${ }^{26} k_{\text {diff }}$ is the rate of diffusion of $\mathrm{OH}$ radical out of the laser overlap region in the supersonic flow. ${ }^{25}$ Eqn (E4) was fitted to the experimental data for the time profile of $\mathrm{OH}$ to give $k_{1}{ }^{\prime}+k_{\text {diff }}$ and the bimolecular rate coefficient $k_{1}{ }^{\prime}$ was determined as the gradient of a plot of $k_{1}{ }^{\prime}+k_{\text {diff }}$ versus $\left[\mathrm{CH}_{3} \mathrm{C}(\mathrm{O}) \mathrm{CH}_{3}\right]$.

The $\mathrm{OH}$ temporal profiles using $t$ - $\mathrm{BuOOH}$ as the $\mathrm{OH}$ precursor were described well by a single exponential decay, and the following function was fitted to the data:

$$
[\mathrm{OH}]=[\mathrm{OH}]_{10} \exp \left(-k_{X}{ }^{\prime} \cdot t\right)
$$

where $[\mathrm{OH}]_{0}$ is the initial concentration of $\mathrm{OH}$ radicals at $t=0$ and $k_{X}{ }^{\prime}$ is the pseudo-first-order rate constant, which, depending on the reaction being studied, is given by either $k_{X}{ }^{\prime}=$ $k_{1}\left[\mathrm{CH}_{3} \mathrm{C}(\mathrm{O}) \mathrm{CH}_{3}\right]+k_{\text {other }}{ }^{\prime}$ or $k_{X}{ }^{\prime}=k_{2}[\mathrm{DME}]+k_{\text {other }}{ }^{\prime}$, where $k_{\text {other }}{ }^{\prime}$ is the rate of loss of $\mathrm{OH}$ by reaction with the precursor and by diffusion out of the observation region. $k_{1}$ and $k_{2}$ were then obtained from a plot of $k_{X}{ }^{\prime}$ versus $\left[\mathrm{CH}_{3} \mathrm{C}(\mathrm{O}) \mathrm{CH}_{3}\right]$ and [DME], respectively.

\section{Theoretical calculations}

Rate coefficients were calculated using the energy grained master equation solver MESMER (Master Equation Solver for Multi Energy well Reactions). ${ }^{24}$ This package is an open source program which solves the chemical master equation for a reaction system in order to obtain phenomenological rate coefficients using an eigenvector/eigenvalue method. Given a potential energy surface with an arbitrary number of intermediates, the coupled differential equations describing both reactive and energy transfer processes are solved allowing the role of the pre-reaction complex to be considered explicitly. In this approach, the phase space of each isomer is lumped into energy grains of a set size and the time evolution of the grain populations are then calculated individually in order to obtain the time evolution for the concentration of each species. A grain size of $50 \mathrm{~cm}^{-1}$ was used, which is sufficiently small for convergence of the calculated rate coefficients above $60 \mathrm{~K}$, although a smaller grain size of $10 \mathrm{~cm}^{-1}$ was used to ensure convergence below $60 \mathrm{~K}$.

Isoenergetic reaction rates between grains of different isomers are calculated using RRKM theory, whilst energy transfer between grains of the same species is modelled using an exponential down model parameterised by an energy independent $\left\langle\Delta E_{\text {down }}\right\rangle$, the average energy transferred in a downward direction upon collision with a bath gas molecule. Once both reactive and collisional transition probabilities have been obtained from the RRKM and exponential down model expressions, the evolution of the grain populations is given by the matrix equation: ${ }^{24}$

$$
\frac{\mathrm{d} \boldsymbol{n}}{\mathrm{d} t}=[\omega(\boldsymbol{P}-\boldsymbol{I})-\boldsymbol{k}] \boldsymbol{n}=\boldsymbol{M n}
$$

where $\boldsymbol{P}$ is a matrix consisting of transition probabilities, $\boldsymbol{I}$ is the identity matrix, $\boldsymbol{k}$ is a diagonal matrix consisting of microcanonical rate coefficients and $\boldsymbol{n}$ is the population vector. The solution of (E6) is then given by:

$$
\boldsymbol{n}(t)=\sum_{i} \boldsymbol{u}_{i} c_{i} \exp \left(\lambda_{i} t\right)
$$

where $\boldsymbol{u}_{i}$ are the eigenvectors with corresponding eigenvalues $\lambda_{i}$, and $c_{i}$ are formed from a product of the inverse of the eigenvalue matrix and the initial population vector. Finally, in order to obtain experimental observables or phenomenological rate coefficients from the master equation calculations, the Bartis Widom method of eigenvalue analysis is used..$^{27-29}$ The master equation calculations have been extended to include a bimolecular source term corresponding to the barrierless association between $\mathrm{OH}$ and either acetone or DME to form the pre-reaction complex (IM1). Microcanonical rate coefficients for the dissociation of the complex back to reactants were determined using an inverse Laplace transform (ILT) of the canonical high pressure limiting rate coefficient, described by the Arrhenius form: ${ }^{30}$

$$
k^{\infty}(T)=A^{\infty}\left(\frac{T}{T^{\infty}}\right)^{n^{\infty}} \mathrm{e}^{\left(\frac{-E^{\infty}}{R T}\right)}
$$

where $A^{\infty}$ is the pre-exponential factor, $E^{\infty}$ is the activation energy in units identical to those of the corresponding stationary 
point energies, and $n^{\infty}$ accounts for any temperature dependence in the observed values of $k^{\infty}(T)$. Microcanonical rate coefficients are subsequently obtained through taking an ILT of this expression.

For both the reactions of $\mathrm{OH}$ with acetone and DME there are already a number of potential energy surfaces in the literature and new surfaces have therefore not been calculated in the current work. For the reaction of $\mathrm{OH}$ with acetone, the surface used by Caralp et al. ${ }^{20}$ was employed, based on calculations by Henon et al. ${ }^{31}$ at the MP2/6-31G**//CCSD/6-311G** level of theory. For the reaction of $\mathrm{OH}$ with $\mathrm{DME}$, the recent surface calculated by Carr et al. ${ }^{32}$ was employed, which used a full coupled cluster with complete basis set extrapolation FCC/ CBS//CCSD/AVDZ level of theory. Further details regarding the optimisations for the geometric parameters and energies of the reactants, complexes, transition states and products are given in Carr et al. ${ }^{32}$

The calculated surfaces for both of these reactions identify more than one saddle point corresponding to transition states (TS2) for hydrogen abstraction. For the reaction of $\mathrm{OH}$ with DME ${ }^{32}$ it was identified that these three separate saddle points were in fact different conformers of one distinct transition state, related by hindered internal rotations. As the emphasis of this work is upon the low temperature behaviour of these reactions, a potential energy surface consisting of a pre-reaction well with one transition state was used for both reactions, with the TS corresponding to the lowest energy $a b$ initio calculated saddle point. In these calculations the final reaction step from IM2, see Fig. 1, to bimolecular products was not considered, with the reaction between IM1 and IM2 being treated as irreversible. It was found that this approximation introduced an error of less than $1 \%$ over the temperature range of our simulations. ${ }^{32}$

In order to calculate the molecular sums and densities of states, rigid rotor and harmonic oscillator approximations were used for all rotations and most vibrational frequencies, respectively. However, there are a number of low frequency vibrational normal modes present in many of the species of both PESs which are better described as hindered or free internal rotations. Hindrance potentials for these modes were calculated using the Gaussian 03 suite of $a b$ initio software ${ }^{33}$ at the MPW1K/6-31+G(2d,2p) level of theory. The rotational potential was then calculated by incrementing the dihedral angle corresponding to rotation about $360^{\circ}$ and at each increment a constrained geometry optimisation was performed. Some of the relaxed scans which incorporated the long-range hydrogen bonded interaction between the $\mathrm{OH}$ and the acetone failed to calculate potentials about a full $360^{\circ}$. Relaxed potentials were instead approximated for these modes through calculating a rigid potential, and then performing a constrained geometry optimisation at the value of the dihedral angle corresponding to the barrier to rotation. The rest of the potential was then scaled by the difference between rigid and relaxed barriers. For each hindered rotational mode a vector corresponding to the torsional coordinates was obtained and such vectors were projected from the Hessian for each species according to the methods described by Sharma et al. ${ }^{34}$ The resulting frequencies, obtained upon diagonalisation of the projected Hessian are then orthogonal to the torsional motions.

As these reactions involve a hydrogen transfer, tunnelling effects are expected to be significant, particularly at low temperatures. Tunnelling transmission coefficients were calculated and the RRKM rate coefficients correspondingly corrected. Tunnelling was treated via two methods, using the imaginary frequency of the transition state calculated from an Eckart potential according to the method of Miller, ${ }^{35}$ and using the semi classical Wentzel-Kramers-Brillouin (WKB) method. ${ }^{36}$ For the latter method, the change in potential energy along the entire reaction path was required and this was obtained by performing an intrinsic reaction co-ordinate (IRC) calculation at the MPW1K/6-31+G(2d,2p) level of theory. ${ }^{33}$ The IRC was subsequently refined through performing projected frequency calculations to obtain the zero point energy at a number of points along the potential and $\operatorname{CCSD}(\mathrm{T}) /$ aug-cc-pVDZ calculations were also performed to obtain higher level electronic energies at these points. This approach together with a WKB tunnelling method gave good agreement with experiment in a previous study of the reaction of $\mathrm{OH}$ with acetone. ${ }^{1}$

The purpose of the modelling is to complement and fit the experimental results, rather than to attempt to calculate the rate coefficients a priori. Given the large amount of experimental data available both from the current work and in previous studies, a number of parameters were varied in order to fit the experimental data through minimising the goodness of fit parameter $\chi^{2}$ :

$$
\chi^{2}=\sum \frac{\left(k_{\mathrm{obs}}-k_{\mathrm{calc}}\right)^{2}}{\sigma_{k_{\mathrm{obs}}}^{2}}
$$

where $k_{\mathrm{obs}}$ are the experimental rate coefficients for the reaction, $k_{\text {calc }}$ are the corresponding master equation calculated rate coefficients and $\sigma_{k_{\mathrm{obs}}}$ are the experimental $(1 \sigma)$ uncertainties. The fitting was carried out using a Levenburg Marquardt algorithm built into the MESMER code. With regards to the barrierless association reaction between $\mathrm{OH}$ and either acetone or DME to form the pre-reaction complex, the high pressure limiting rate coefficient, $k_{\infty}$, given by (E8) above, requires the $A_{\infty}$ parameter for the ILT calculations, and any knowledge of the temperature dependence of the reaction. Formally, a dipole-dipole interaction would be expected to display a weak negative temperature dependence, ${ }^{37}$ but this has been neglected in the current work and the ILT $n_{\infty}$ parameter in (E8) is set to 0 . In addition, the calculated rate coefficients were found in both cases to be very sensitive to the energies of IM1 and TS2, and these parameters were also varied. The energy transfer parameter $\left\langle\Delta E_{\text {down }}\right\rangle$ was found to be too strongly correlated with the well depth of IM1 to allow both parameters to be floated, and so for reactions of $\mathrm{OH}$ with both acetone and DME a fixed value of $\left\langle\Delta E_{\text {down }}\right\rangle$ of $300 \mathrm{~cm}^{-1}$ was assigned. Although this value for $\left\langle\Delta E_{\text {down }}\right\rangle$ is toward the upper end of values determined for similar systems with a $\mathrm{N}_{2}$ bath gas, ${ }^{38}$ it was found that smaller values of $\left\langle\Delta E_{\text {down }}\right\rangle$ resulted in a larger IM1 binding energy due to this strong correlation. This fitting approach has been successfully carried out previously for the reaction of $\mathrm{OH}$ with 
DME at higher temperatures ${ }^{32}$ and was found to be an effective way of exploring the mechanism of a reaction through the sensitivities of different parameters.

\section{Results}

\subsection{Measurement of rate coefficients}

Rate coefficients for the reactions of $\mathrm{OH}$ with acetone and DME were experimentally measured for temperatures between 63 and $148 \mathrm{~K}$ and for total gas densities ranging from $3.2 \times 10^{16}$ to $2.6 \times 10^{17}$ molecule $\mathrm{cm}^{-3}$, and are listed in Tables 1 and 2 for acetone and DME, respectively. For acetone, the rate coefficients are plotted as a function of temperature and total density (for 80 and $146 \mathrm{~K}$ ) in Fig. 2 and 4, respectively, with the corresponding data for DME plotted in Fig. 3 and 5 respectively.

The results clearly demonstrate a dramatic, inverse temperature dependence of the rate coefficients of both reactions for temperatures below $200 \mathrm{~K}$, consistent with our previous, initial study over a smaller range of temperature (86-112 $\mathrm{K}$ for acetone, 93-112 $\mathrm{K}$ for DME, both at a single density). ${ }^{23} \mathrm{We}$ attribute the enhancement in the rate coefficients for $\mathrm{OH}$ loss to the presence of the pre-reaction complex (IM1) which is calculated to have a significant binding energy for both systems. $^{31,32,39,40}$ We postulate that this complex enhances the rate coefficient either through providing a termolecular stabilisation channel or through enhancing the bimolecular abstraction channel by quantum mechanical tunnelling at low temperatures. ${ }^{23}$ The data recorded in this study over a wider range of temperature and pressure will enable the contribution from each mechanism to be investigated, which we did not report in our previous publication.

Table 1 Rate coefficients for the reaction of $\mathrm{OH}$ with acetone measured using a pulsed Laval nozzle apparatus in combination with PLP-LIF detection of $\mathrm{OH}$. The errors bars for the temperature and density represent the $1 \sigma$ uncertainties in the impact pressure measurements, and the error bars for the rate coefficients are the $95 \%$ confidence intervals in the linear fits of the pseudo-first-order coefficients plotted as a function of reagent density, together with systematic errors

\begin{tabular}{|c|c|c|c|}
\hline $\begin{array}{l}\text { Temperature/ } \\
\mathrm{K}\end{array}$ & $\begin{array}{l}\mathrm{OH} / \mathrm{OD} \\
\text { precursor }\end{array}$ & $\begin{array}{l}\text { Flow density } / \\
10^{16} \text { molecule } \mathrm{cm}^{-3}\end{array}$ & $\begin{array}{l}\text { Rate coefficient, } k_{1} / \\
\text { molecule }^{-1} \mathrm{~cm}^{3} \mathrm{~s}^{-1}\end{array}$ \\
\hline $79 \pm 3$ & Acetyl $/ \mathrm{O}_{2}$ & $17 \pm 2$ & $(1.0 \pm 0.1) \times 10^{-10 a}$ \\
\hline $82 \pm 4$ & Acetyl $/ \mathrm{O}_{2}$ & $3.2 \pm 0.6$ & $(3.7 \pm 0.4) \times 10^{-11}$ \\
\hline $82 \pm 4$ & Acetyl $/ \mathrm{O}_{2}$ & $5.1 \pm 0.5$ & $(5.9 \pm 0.6) \times 10^{-11 a}$ \\
\hline $86 \pm 3$ & Acetyl $/ \mathrm{O}_{2}$ & $6.5 \pm 0.6$ & $(5.6 \pm 0.6) \times 10^{-11 b}$ \\
\hline $83 \pm 3$ & Acetyl $/ \mathrm{O}_{2}$ & $8.9 \pm 0.6$ & $(7.6 \pm 0.8) \times 10^{-11}$ \\
\hline $81 \pm 4$ & Acetyl $/ \mathrm{O}_{2}$ & $13 \pm 2$ & $(8.9 \pm 0.9) \times 10^{-11}$ \\
\hline $93 \pm 4$ & Acetyl $/ \mathrm{O}_{2}$ & $6.5 \pm 0.5$ & $(5.6 \pm 0.8) \times 10^{-11 a}$ \\
\hline $112 \pm 9$ & Acetyl $/ \mathrm{O}_{2}$ & $6 \pm 1$ & $(5 \pm 1) \times 10^{-12 a, b}$ \\
\hline $138 \pm 9$ & $t$-BuOOH & $8 \pm 1$ & $(1.3 \pm 0.9) \times 10^{-12 a}$ \\
\hline $133 \pm 10$ & $t$-BuOOH & $13 \pm 3$ & $(2.8 \pm 0.6) \times 10^{-12}$ \\
\hline $148 \pm 15$ & $t$-BuOOH & $10 \pm 1$ & $(1.6 \pm 0.8) \times 10^{-12}$ \\
\hline $143 \pm 15$ & $t$-BuOOH & $18 \pm 2$ & $(2.4 \pm 1.1) \times 10^{-12}$ \\
\hline $146 \pm 15$ & $t$-BuOOH & $26 \pm 4$ & $(3.5 \pm 0.7) \times 10^{-12}$ \\
\hline $146 \pm 15$ & Acetyl $/ \mathrm{O}_{2}$ & $26 \pm 4$ & $(3 \pm 1) \times 10^{-12}$ \\
\hline $93 \pm 4$ & $d$-Acetyl $/ \mathrm{O}_{2}$ & $6.5 \pm 0.5$ & $(6.8 \pm 0.7) \times 10^{-11 c}$ \\
\hline $146 \pm 15$ & $d$-Acetyl $/ \mathrm{O}_{2}$ & $26 \pm 4$ & $(1.4 \pm 1.3) \times 10^{-12 c}$ \\
\hline
\end{tabular}

${ }^{a}$ Data presented in Fig. 2. ${ }^{b}$ Data previously published in Shannon et al. ${ }^{1}{ }^{c}$ Reaction under study is $\mathrm{OD}+\mathrm{CD}_{3} \mathrm{COCD}_{3} \rightarrow \mathrm{CD}_{3} \mathrm{COCD}_{2}+\mathrm{D}_{2} \mathrm{O}$.
Table 2 Rate coefficients for the reaction of $\mathrm{OH}+$ DME measured using a pulsed Laval nozzle apparatus in combination with PLP-LIF detection of $\mathrm{OH}$. The errors bars for the temperature and density represent the $1 \sigma$ uncertainties in the impact pressure measurements, and the error bars for the rate coefficients are the $95 \%$ confidence intervals in the linear fits of the pseudo-first-order coefficients plotted as a function of reagent density, together with systematic errors

\begin{tabular}{lll}
\hline $\begin{array}{l}\text { Temperature/ } \\
\mathrm{K}\end{array}$ & $\begin{array}{l}\text { Flow density/ } \\
10^{16} \text { molecule } \mathrm{cm}^{-3}\end{array}$ & $\begin{array}{l}\text { Rate coefficient, } k_{2} / \\
\text { molecule }^{-1} \mathrm{~cm}^{3} \mathrm{~s}^{-1}\end{array}$ \\
\hline $63 \pm 2$ & $3.4 \pm 0.3$ & $(1.7 \pm 0.1) \times 10^{-11 a}$ \\
$83 \pm 3$ & $6.1 \pm 0.5$ & $(8 \pm 1) \times 10^{-12 a}, b$ \\
$81 \pm 4$ & $13 \pm 2$ & $(1.6 \pm 0.3) \times 10^{-11}$ \\
$83 \pm 3$ & $8.9 \pm 0.6$ & $(9 \pm 1) \times 10^{-12}$ \\
$79 \pm 3$ & $17 \pm 2$ & $(7.9 \pm 0.2) \times 10^{-11}$ \\
$93 \pm 4$ & $6.5 \pm 0.5$ & $(4.1 \pm 1.2) \times 10^{-12 a}, b$ \\
$110 \pm 8$ & $9.1 \pm 1.4$ & $(3.1 \pm 0.5) \times 10^{-12 a}$ \\
$138 \pm 9$ & $8 \pm 1$ & $(3.0 \pm 0.4) \times 10^{-12}$ \\
$133 \pm 10$ & $13 \pm 3$ &
\end{tabular}

${ }^{a}$ Data presented in Fig. $3 .{ }^{b}$ Data previously published in Shannon et al. ${ }^{1}$

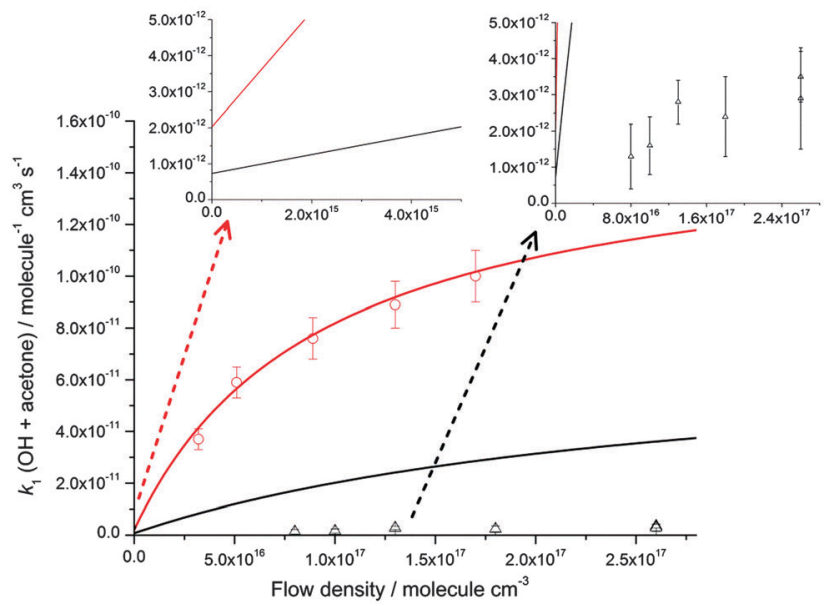

Fig. 4 Pressure dependence of the rate coefficients for the reaction of $\mathrm{OH}$ with acetone at $\sim 80 \mathrm{~K}$ (red circles, the temperature varies between $79 \pm 3 \mathrm{~K}$ and $83 \pm 3 \mathrm{~K}$ for these points) and $\sim 140 \mathrm{~K}$ (black triangles, the temperature varies between $133 \pm 10 \mathrm{~K}$ and $148 \pm 15 \mathrm{~K}$ for these points) plotted alongside the MESMER master equation calculations at $80 \mathrm{~K}$ (red line) and $140 \mathrm{~K}$ (black line). The insets show non-zero intercepts calculated using MESMER at both 80 and $140 \mathrm{~K}$ and a vertically expanded version of the $140 \mathrm{~K}$ experimental data. The experimental error bars are as shown in Table 1.

To explore the contribution from tunnelling, rate coefficients were measured for the reaction of OD with acetone- $\mathrm{d}_{6}$ at 93 and $146 \mathrm{~K}$ :

$$
\mathrm{OD}+\mathrm{CD}_{3} \mathrm{C}(\mathrm{O}) \mathrm{CD}_{3} \rightarrow \mathrm{CD}_{3} \mathrm{C}(\mathrm{O}) \mathrm{CD}_{2}+\mathrm{D}_{2} \mathrm{O}
$$

This system was chosen in preference to the reaction of $\mathrm{OH}$ with acetone- $\mathrm{d}_{6}$ or OD with undeuterated acetone since the acetone- $\mathrm{d}_{6}$ could be used both as an OD precursor in the presence of $\mathrm{O}_{2}$, via the reaction of $\mathrm{CD}_{3} \mathrm{CO}+\mathrm{O}_{2}$, and the reactant. If tunnelling were the dominant mechanism, one would expect its rate to be significantly slower for a D-atom transfer compared to a H-atom. However, as can be seen in Table 1, 


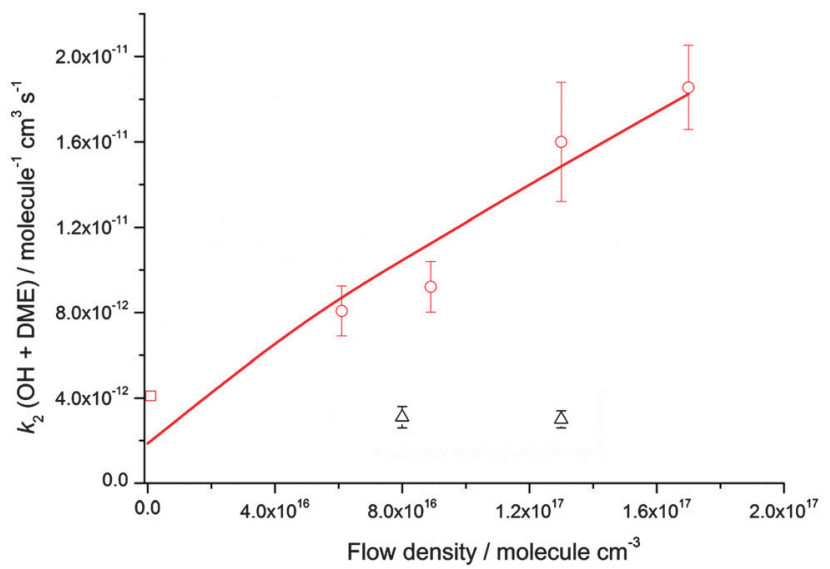

Fig. 5 MESMER master equation calculations (red line, $80 \mathrm{~K}$ ) together with experimentally determined values of $k_{2}(\mathrm{OH}+\mathrm{DME})$ for a range of different total gas densities at a temperature of $\sim 80 \mathrm{~K}$ (red circles, the temperature varies between $79 \pm 3 \mathrm{~K}$ and $83 \pm 3 \mathrm{~K}$ ) and $140 \mathrm{~K}$ (black triangles, the temperature varies between $133 \pm 10 \mathrm{~K}$ and $138 \pm 9 \mathrm{~K})$. The rate coefficient shown at zero pressure (red square) is estimated using the $\mathrm{OH}$ recycling experiments in the presence of $\mathrm{O}_{2}$ performed at $93 \pm 4 \mathrm{~K}$, see text for details. The experimental errors bars are as given in Table 2 .

at $93 \mathrm{~K}$, and for very similar overall densities, the rate coefficients for (R1) and (R6) are similar (within error of each other), providing evidence that tunnelling through TS2 is not the dominant channel. At $146 \mathrm{~K}$, the rate coefficient for (R6) is about a factor of 2 lower than for (R1), again for a very similar density, but the rate coefficients are subject to a considerably larger error (almost $100 \%$ for the case of the deuterated reaction (R6)), because the magnitude of the rate coefficient is close to the limit of what can be measured in the limited timewindow of the pulsed Laval method. It is hence difficult to be certain of any change with deuteration at this temperature. No deuteration experiments were attempted for the reaction of OH with DME.

These results suggest that for the reaction of $\mathrm{OH}$ with acetone, the dominant influence for the large enhancement in the rate coefficient observed below $200 \mathrm{~K}$ is a LindemannHinshelwood type mechanism in which the $\mathrm{OH}$-acetone association complex (IM1) is being formed as a stabilised product. If such a mechanism were operating, it would be expected that the overall rate coefficient for $\mathrm{OH}$ loss $\left(k_{1}\right)$ would be proportional to the number of deactivating collisions between the $\mathrm{N}_{2}$ bath gas molecules and energised IM1 species, and hence the total $\mathrm{N}_{2}$ number density. Using two Laval nozzles, $k_{1}$ was measured at a variety of $\left[\mathrm{N}_{2}\right]$ for temperatures between $79-86 \mathrm{~K}$ and also $133-146 \mathrm{~K}$, as shown in Fig. 4. There is a small variation in temperature as the total gas density changes for a given nozzle. At $\sim 80 \mathrm{~K}$, there is an increase in the rate coefficient with $\left[\mathrm{N}_{2}\right]$, consistent with the Lindemann-Hinshelwood mechanism for stabilisation of the $\mathrm{OH}$-acetone complex. However, at $\sim 140 \mathrm{~K}$ the rate coefficient is less dependent upon pressure, although it must be noted that the uncertainties are considerably larger owing to the smaller rate coefficients. For the temperatures around $140 \mathrm{~K}$, it was not possible to measure $k_{1}$ at the lower densities used at $\sim 80 \mathrm{~K}$, as the magnitude of $k_{1}$ was already approaching $\sim 1 \times 10^{-12} \mathrm{~cm}^{3}$ molecule ${ }^{-1} \mathrm{~s}^{-1}$, which is the lower limit for this technique, owing to the more limited $(\sim 100 \mu \mathrm{s}$ compared to $\sim 250 \mu \mathrm{s}$ at $80 \mathrm{~K}$ ) reaction time and amount of acetone which can be added and still maintain a stable flow. However, at $\sim 140 \mathrm{~K}$, the data suggest that collisional stabilisation of the complex is unable to compete with its re-dissociation to reactants, and the measured rate coefficients (which are considerably smaller) are dominated by a pressure independent mechanism, such as tunnelling.

Rate coefficients for the reaction of $\mathrm{OH}$ with DME, $k_{2}$, were also measured at four different total $\left[\mathrm{N}_{2}\right]$ densities for the same nozzle for temperatures between $79-83 \mathrm{~K}$, and are displayed in Fig. 5. $k_{2}$ is seen to increase significantly with $\left[\mathrm{N}_{2}\right]$, with the reaction appearing to be closer to the linear, low density limiting regime, compared to the reaction of $\mathrm{OH}$ with acetone (Fig. 4) at the same temperature.

The dependence of both $k_{1}$ and $k_{2}$ upon $\left[\mathrm{N}_{2}\right]$ at $\sim 80 \mathrm{~K}$ is evidence that in both cases, a stabilisation mechanism is dominating at this temperature and for the density range of the experiments performed. However, this does not exclude the possibility of a tunnelling mechanism dominating at even lower temperatures and/or densities. Indeed, inspection of Fig. 4 and 5 suggest that extrapolation to zero pressure may yield non-zero rate coefficients supportive of the presence of a bimolecular channel such as tunnelling. In a recent study of the reaction of $\mathrm{OH}$ with methanol at $\sim 80 \mathrm{~K},{ }^{5}$ the overall rate coefficient was observed to be independent of pressure, consistent with a tunnelling mechanism and supported by theoretical calculations. The tunnelling mechanism was further supported by observation of the $\mathrm{CH}_{3} \mathrm{O}$ radical product using LIF, consistent with a high calculated yield for this product under these conditions.

For the reaction of $\mathrm{OH}$ with DME:

$$
\mathrm{OH}+\mathrm{CH}_{3} \mathrm{OCH}_{3} \stackrel{k_{2}}{\rightarrow} \mathrm{CH}_{3} \mathrm{OCH}_{2}+\mathrm{H}_{2} \mathrm{O}
$$

there is a convenient experimental method to probe whether the methoxymethyl radical abstraction product, $\mathrm{CH}_{3} \mathrm{OCH}_{2}$, is being formed. The presence of $\mathrm{CH}_{3} \mathrm{OCH}_{2}$ provides evidence that quantum mechanical tunnelling is making a contribution to the overall reaction mechanism at very low temperatures where there is insufficient energy to surmount the activation barrier. In the presence of $\mathrm{O}_{2}$, if the $\mathrm{CH}_{3} \mathrm{OCH}_{2}$ product is formed, it can react further to form an energised peroxy radical: ${ }^{41,55}$

$$
\mathrm{CH}_{3} \mathrm{OCH}_{2}+\mathrm{O}_{2} \rightarrow \mathrm{CH}_{3} \mathrm{OCH}_{2} \mathrm{O}_{2}{ }^{*}
$$

which can either re-dissociate back to reactants, be stabilised by collisions to form the relatively stable peroxy radical $\mathrm{CH}_{3} \mathrm{OCH}_{2} \mathrm{O}_{2}$, (R8a) or dissociate to regenerate $\mathrm{OH}$ and two molecules of formaldehyde (R8b):

$$
\begin{array}{r}
\mathrm{CH}_{3} \mathrm{OCH}_{2} \mathrm{O}_{2}{ }^{*}+\mathrm{M} \stackrel{k_{8 a}}{\longrightarrow} \mathrm{CH}_{3} \mathrm{OCH}_{2} \mathrm{O}_{2}+\mathrm{M} \\
\mathrm{CH}_{3} \mathrm{OCH}_{2} \mathrm{O}_{2}{ }^{*} \stackrel{k_{8 b}}{\longrightarrow} \mathrm{OH}+2 \mathrm{H}_{2} \mathrm{CO}
\end{array}
$$

via the formation of $\mathrm{CH}_{2} \mathrm{OCH}_{2} \mathrm{OOH}^{*}$ following an internal $\mathrm{H}$ abstraction. ${ }^{41} \mathrm{OH}$ recycling of this type has been seen in a 
number of reactions of $\mathrm{OH}$ in the presence of $\mathrm{O}_{2} \cdot{ }^{32,42,43}$ As long as $\left(k_{8 \mathrm{a}}+k_{8 \mathrm{~b}}\right)\left[\mathrm{O}_{2}\right] \gg k_{2}\left[\mathrm{CH}_{3} \mathrm{OCH}_{3}\right]$ through the addition of sufficiently high $\mathrm{O}_{2}$, and $\left[\mathrm{CH}_{3} \mathrm{OCH}_{3}\right] \gg[\mathrm{OH}]$, then regeneration via reaction (R8b) is rapid, and the pseudo-first-order rate coefficients for the overall loss of $\mathrm{OH}$ are given by: ${ }^{32,41}$

$$
\begin{gathered}
k_{2}{ }^{\prime}\left(\text { no } \mathrm{O}_{2} \text { present }\right)=k_{2}\left[\mathrm{CH}_{3} \mathrm{OCH}_{3}\right] \\
k_{2}{ }^{\prime}\left(\mathrm{O}_{2} \text { present }\right)=k_{2}(1-\alpha)\left[\mathrm{CH}_{3} \mathrm{OCH}_{3}\right]
\end{gathered}
$$

where $\alpha$ is the absolute yield of $\mathrm{OH}$ that is recycled via reaction (R8b) following the formation of $\mathrm{CH}_{3} \mathrm{OCH}_{2}$ in the presence of $\mathrm{O}_{2}$, which is given by: ${ }^{41}$

$$
\alpha=1-\left(\frac{k_{2}\left(\mathrm{O}_{2} \text { present }\right)}{k_{2}\left(\text { no } \mathrm{O}_{2} \text { present }\right)}\right)
$$

At $93 \mathrm{~K}$ experiments were performed with $20 \%$ of the bath gas comprising $\mathrm{O}_{2}$, and the variation of $k_{2}{ }^{\prime}$ with $\left[\mathrm{CH}_{3} \mathrm{OCH}_{3}\right]$ both with and without the addition of $\mathrm{O}_{2}$ are shown in Fig. 6, for a total density in $\mathrm{N}_{2}$ of $6.5 \times 10^{16}$ molecule $\mathrm{cm}^{-3}$. A clear reduction in the gradient is seen in the presence of $\mathrm{O}_{2}$, with $k_{2}(1-\alpha)\left(\mathrm{O}_{2}\right.$ present $)=(8.0 \pm 0.8) \times 10^{-12}$ molecule ${ }^{-1} \mathrm{~cm}^{3} \mathrm{~s}^{-1}$ and $k_{2}=(1.7 \pm 0.1) \times 10^{-11}$ molecule ${ }^{-1} \mathrm{~cm}^{3} \mathrm{~s}^{-1}$ (no $\mathrm{O}_{2}$ present). These results provide evidence that the $\mathrm{CH}_{3} \mathrm{OCH}_{2}$ radical is indeed formed as a direct product from the reaction of $\mathrm{OH}$ with DME at $93 \mathrm{~K}$, for example via quantum mechanical tunnelling. Using the rate coefficients obtained in the presence and absence of $\mathrm{O}_{2}$, a yield of $\mathrm{OH}$ of $\alpha=0.53_{-0.08}^{+0.07}$ is obtained. If all the $\mathrm{CH}_{3} \mathrm{OCH}_{2}$ radicals were being recycled to form $\mathrm{OH}$ via reaction (R8b), then the yield of $\mathrm{CH}_{3} \mathrm{OCH}_{2}$ product from the abstraction reaction of $\mathrm{OH}$ with DME is about $50 \%$. However, this is only a lower limit to the yield of $\mathrm{CH}_{3} \mathrm{OCH}_{2}$, as some of the initially formed $\mathrm{CH}_{3} \mathrm{OCH}_{2} \mathrm{O}_{2}{ }^{*}$ will be collisionally stabilised

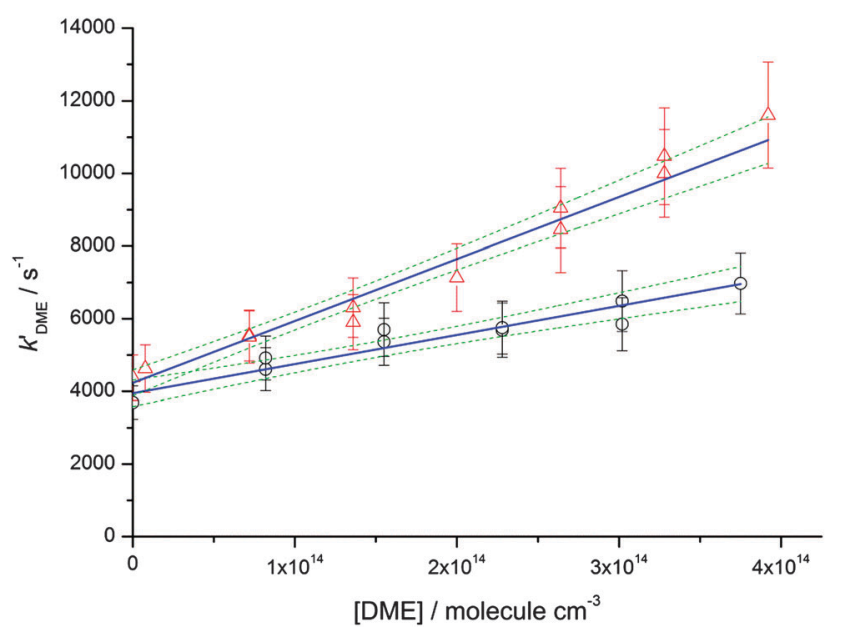

Fig. 6 Variation of the pseudo-first-order rate coefficient for the loss of $\mathrm{OH}$ radicals as a function of the density of DME in the presence of $\left[\mathrm{O}_{2}\right]=$ $3 \times 10^{15}$ molecule $\mathrm{cm}^{-3}$ (black open circles) and without $\mathrm{O}_{2}$ (open red triangles). The blue full lines are the weighted linear fits to the data, and the dashed green lines represent upper and lower 95\% confidence limits. The gradients of the blue lines yield bimolecular rate coefficients $k_{2}=$ (8.0 \pm 0.8$) \times 10^{-12}$ molecule $\mathrm{cm}^{3} \mathrm{~s}^{-1}$ (in the presence of $\mathrm{O}_{2}$ ) and $(1.7 \pm 0.1) \times 10^{-11}$ molecule $\mathrm{cm}^{3} \mathrm{~s}^{-1}$ (without $\mathrm{O}_{2}$ ). The total density of $\mathrm{N}_{2}=(6.5 \pm 0.5) \times 10^{16}$ molecule $\mathrm{cm}^{-3}$. to the peroxy radical $\mathrm{CH}_{3} \mathrm{OCH}_{2} \mathrm{O}_{2}$ via reaction ( $\mathrm{R} 8 \mathrm{a}$ ), and $\mathrm{OH}$ is therefore not recycled. If we assume this lower limit, namely that $\mathrm{CH}_{3} \mathrm{OCH}_{2} \mathrm{O}_{2}{ }^{*}$ is not stabilized by collisions (the association reaction of $\mathrm{CH}_{3} \mathrm{OCH}_{2}$ with $\mathrm{O}_{2}$ is in the low-pressure regime), then multiplying the overall rate coefficient by the yield allows a lower limit for the rate coefficient in the limit of zero pressure to be determined as $0.53 \times 7.7 \times 10^{-12}=$ $4.1 \times 10^{-12} \mathrm{~cm}^{3}$ molecule ${ }^{-1} \mathrm{~s}^{-1} .{ }^{44}$ This value has been added to Fig. 5 at zero pressure. At $93 \mathrm{~K}$ we did not perform the addition of $\mathrm{O}_{2}$ experiments as a function of total density of $\mathrm{N}_{2}$, and the branching ratio $\frac{k_{8 \mathrm{~b}}}{k_{8 \mathrm{a}}+k_{8 \mathrm{~b}}}$ to form $\mathrm{OH}$ from $\mathrm{CH}_{3} \mathrm{OCH}_{2} \mathrm{O}_{2}{ }^{*}$ is unknown experimentally under our conditions. Experiments performed at $80 \mathrm{~K}$ using the same methodology gave $\mathrm{OH}$ yields of $0.14 \pm 0.08,0.13 \pm 0.12$ and $0.10 \pm 0.09$ for total densities of 5.2, 8.7 and $16.4 \times 10^{16}$ molecule $\mathrm{cm}^{-3}\left(\mathrm{~N}_{2}\right)$, respectively, but the large uncertainties preclude any quantitative discussion of these yields. The significant yield of $\mathrm{CH}_{3} \mathrm{OCH}_{2}$ via a tunnelling mechanism is consistent with the intercept of Fig. 5 from data measured at higher pressures which shows the pressure dependent contribution to the overall rate coefficient is significant.

\subsection{MESMER calculation of rate coefficients}

In order to complement the experimental results, rate coefficients were calculated for both reactions of $\mathrm{OH}$ with acetone and DME using the master equation software package MESMER. ${ }^{24}$ As described in Section 3, given the large amount of experimental data available in this study, a fitting methodology was employed in order to yield information about the kinetic parameters and mechanisms of the reactions. The effect of treating torsional modes explicitly was considered, with fits to experimental data carried out considering the vibrations as harmonic oscillators, and as hindered rotors, and the results compared.

Due to the large number of experimental studies which have measured the rate coefficient $k_{1}$ for the reaction of $\mathrm{OH}$ with acetone at temperatures above $\sim 200 \mathrm{~K}$, only a subset of the available data set was used for the fitting process, as the focus of this current work is the lower temperature regime. The studies by both Gierczak et al. ${ }^{45}$ and Yamada et al. ${ }^{39}$ contained sufficient data points to span the $200-800 \mathrm{~K}$ temperature range, with no more than $100 \mathrm{~K}$ between each point. A total of 13 experimental rate coefficients from previous studies and the values from this work, were used to obtain the best-fit values of the energies of TS2, IM1 (see Fig. 1) as well as vibrational frequencies (including imaginary values corresponding to the tunnelling coordinate), and the $A$ factor used in the ILT procedure. The optimised parameters from fitting to the experimental kinetic data are given in Table 3, together with ab initio calculations from previous studies. ${ }^{31,39,40}$ It is clear from inspection of Table 3 that the harmonic oscillator and hindered rotor models give significantly different results. Considering the energy of TS2 (OH/acetone reaction), the harmonic oscillator only model yields a value of $3.6 \mathrm{~kJ} \mathrm{~mol}^{-1}$, which is in relatively good agreement with the $a b$ initio energy of $6.1 \mathrm{~kJ} \mathrm{~mol}^{-1}$ calculated by Yamada et $a l^{39}$ at the CBS-QB3 level of theory. 
Table 3 Optimum MESMER parameters from performing a Levenburg Marquardt fitting procedure to minimise the goodness of fit parameter, $\chi^{2}$, between the experimental rate coefficient data and theory. The columns marked $a, b$ and $c$ give the $a b$ intio calculated values from Henon et al., 31 Vandenberk et al. ${ }^{40}$ and Yamada et al., ${ }^{39}$ respectively. For the definition of TS2 and IM1 see Fig. 1

\begin{tabular}{|c|c|c|c|c|c|}
\hline Parameter & Harmonic oscillator & Hindered rotor & $a$ & $b$ & $c$ \\
\hline Energy of $\mathrm{TS} 2 / \mathrm{kJ} \mathrm{mol}^{-1}$ & 3.6 & 13.0 & 16.7 & 14.6 & 6.1 \\
\hline Well depth energy of IM1/kJ mol${ }^{-1}$ & -38.1 & -29.4 & -18.4 & -22.2 & -20.1 \\
\hline Imaginary frequency of tunnelling mode $/ \mathrm{cm}^{-1}$ & -541 & -1116 & -1250 & -1107 & -1293 \\
\hline
\end{tabular}

This finding is in agreement with a previous master equation study by Caralp et al. $^{20}$ in which it was found necessary to use the barrier height obtained from the fits to experimental data rather than the larger barrier heights of 14.6 and $16.7 \mathrm{~kJ} \mathrm{~mol}^{-1}$ at the MP2/6-31G**//CCSD(T)/6-311G and B3LYP/6-311++G(d,p)// $\operatorname{CCSD}(\mathrm{T}) / 6-311++\mathrm{G}(\mathrm{d}, \mathrm{p})$ levels of theory calculated by Henon et $a .^{31}$ and Vandenberk et al., ${ }^{40}$ respectively. However, by treating some of the lower frequency vibrations as hindered rotations, the fitted barrier height is $13.0 \mathrm{~kJ} \mathrm{~mol}^{-1}$, in better agreement with the $\operatorname{CCSD}(\mathrm{T})$ calculations.

As seen from Table 3, again for the reaction of $\mathrm{OH}$ with acetone, both the harmonic oscillator and the hindered rotor models require the well depth for IM1 to be larger relative to the $a b$ initio results in order to achieve the best fit with the experimental data. The well depth returned from the fits using the more rigorous hindered rotor model is closer to the ab initio well depths than its harmonic oscillator model counterpart. For both treatments of the vibrational modes, the amount by which the well depth of IM1 has to be increased to give the best fit with the experimental data is likely to be outside the error of the $a b$ initio calculations. A more rigorous treatment of the anharmonic low frequency bending modes of IM1 would likely increase further the density of states of IM1 relative to the separated $\mathrm{OH}+$ acetone reactants, which would in turn reduce the rate coefficient for the re-dissociation of IM1 back to reactants. The well depth of IM1 that is required to give the best fit with the experimental data would then be closer to the $a b$ initio values.

The rate coefficients calculated at zero pressure using MESMER with the hindered rotor model fits for the reaction of $\mathrm{OH}$ with acetone are shown as a function of temperature in Fig. 7, using both the Eckart and WKB methods of tunnelling, and a calculation without a tunnelling correction. As the experimental data above $200 \mathrm{~K}$ are in the low pressure limit, and the data obtained in this study have been shown to be in the fall-off regime, the experimental data obtained in this work are not plotted in Fig. 7. It can be seen that all treatments calculate the rate coefficients as increasing rapidly at very low temperatures, owing to the dominant role played by collisional stabilisation of the complex under those conditions. Indeed at the temperatures for the Laval experiments, the calculated rate coefficients for the different approaches are similar. However, for the experimental data at 200-400 K, the predicted behaviour is quite different. Using the Eckart model with a fitted imaginary frequency, good agreement can be achieved between experiment and theory over a wide range of temperatures despite the

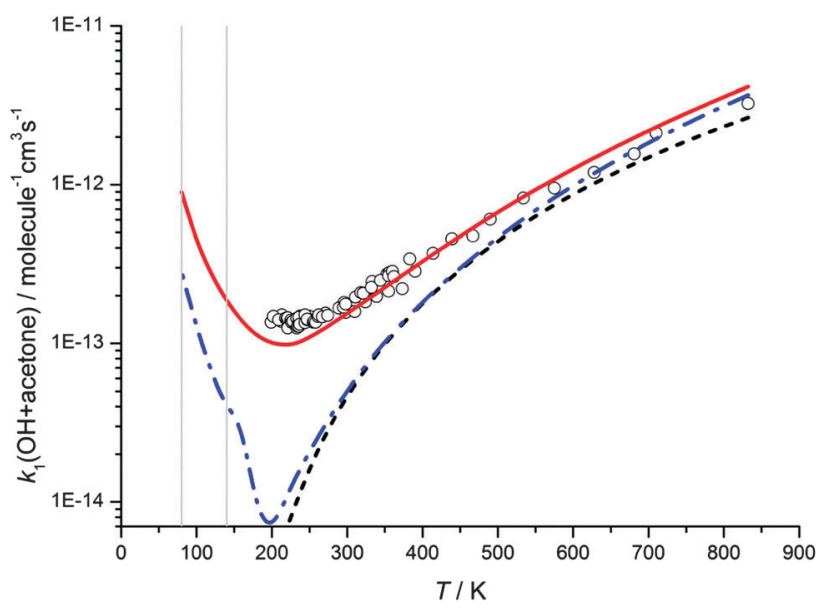

Fig. 7 MESMER master equation calculated rate coefficients for $k_{1}(\mathrm{OH}+$ acetone) versus temperature plotted alongside experimental data currently in the literature (references as for Fig. 2). The red full and blue dot/ dash lines show master equation calculations from the hindered rotor model with transmission coefficients calculated using the Eckart and the WKB methods, respectively, whilst the black dashed line shows calculated rate coefficients without including a tunnelling correction. All MESMER master equation calculations are for the limit of zero pressure. The grey vertical lines are positioned at 80 and $140 \mathrm{~K}$ to aid comparison of the MESMER predicted rate coefficients with the experimentally obtained values listed in Table 1, which were measured at a range of pressures.

larger abstraction barrier height $\left(13.0 \mathrm{~kJ} \mathrm{~mol}^{-1}\right)$. However, from Fig. 7 it can be observed that the WKB tunnelling approach, which is similar in methodology to that used by Caralp et al., ${ }^{20}$ for this reaction, significantly underestimates the experimental rate coefficients in the $200-400 \mathrm{~K}$ region. If no tunnelling correction is included, the experimental rate coefficients are under-estimated by an even greater amount in this region. The reasons for the poor agreement between experiment and theory using the WKB tunnelling method are not clear. Whilst the Eckart approach is more rudimentary, varying the imaginary frequency gives this method a certain degree of flexibility which could be accommodating any limitations in either the potential used for the WKB calculations or the lack of consideration of tunnelling paths orthogonal to the minimum energy path.

The pressure dependence of the rate coefficients for the reaction of $\mathrm{OH}$ with acetone is well described by the master equation calculations at $\sim 80 \mathrm{~K}$, as shown in Fig. 4 , and although predicting a pressure dependence at $140 \mathrm{~K}$, overestimates the magnitude of the rate coefficient significantly. The lack of agreement at $140 \mathrm{~K}$ is unclear, but may be due to an 
incorrect energy dependence of the density of states, which will have more impact in the calculations at higher energies. Another, more speculative reason may be that at $140 \mathrm{~K}$ the system is not as well described using statistical rate theory. The lifetime of the collision complex becomes shorter at higher temperatures, and consequently there might be insufficient time for complete intramolecular vibrational relaxation within the complex which is a central tenet of statistical rate theory, leading to a deviation between experiment and theory.

The master equation calculations show a non-zero $y$-axis intercept in the rate coefficient at zero pressure, corresponding to a pressure independent reaction channel to form products due to tunnelling. The gas densities experienced in molecular clouds are extremely low (typically $10^{2}-10^{4}$ molecule $\mathrm{cm}^{-3}$ ), ${ }^{46}$ and hence the pressure independent channels are the relevant ones to explore the chemistry of these regions. In order to explore the temperature dependence of the pressure independent channel, master equation calculations were performed at a total density of $1 \times 10^{6}$ molecule $\mathrm{cm}^{-3}$ and down to $10 \mathrm{~K}$, as shown in Fig. 8. Calculations were performed using both the Eckhart and WKB treatments of tunnelling, and at $10 \mathrm{~K}$ the predicted rate coefficients are both more than 100 times greater than the room temperature rate coefficient.

The calculated rate coefficients for the reaction of $\mathrm{OH}$ with DME as a function of temperature and pressure are shown in Fig. 5 and 9, respectively. Extensive master equation calculations using MESMER have recently been performed ${ }^{32}$ for this reaction in the range $T=200-1400 \mathrm{~K}$ in order to obtain an optimised set of fitted geometric and energetic parameters that best describe the kinetic data obtained in that study and also extensively reported in the literature at higher temperatures. ${ }^{47-51}$ The calculations showed the presence of a pre-reaction complex with a binding energy of $\sim 22 \mathrm{~kJ} \mathrm{~mol}^{-1}$, but no pressure dependence was experimentally observed, hence providing no direct evidence for stabilisation into the complex influencing the

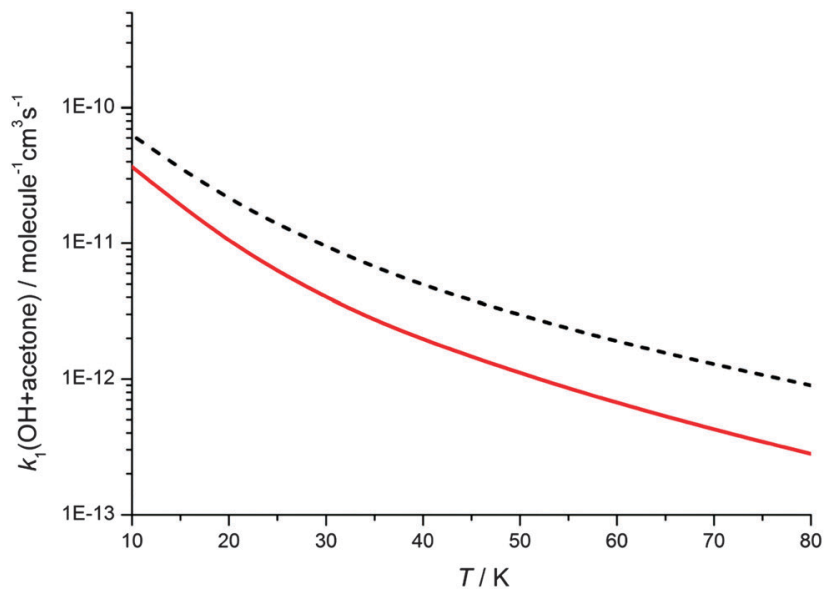

Fig. 8 Rate coefficients $k_{1}(\mathrm{OH}+$ acetone $)$ calculated at a total density of $1 \times 10^{6}$ molecule $\mathrm{cm}^{-3}$ using MESMER. The black dashed line shows calculated rate coefficients using transmission coefficients from the Eckart treatment of tunnelling, whilst the solid red line uses transmission coefficients from the WKB method. See text for details.

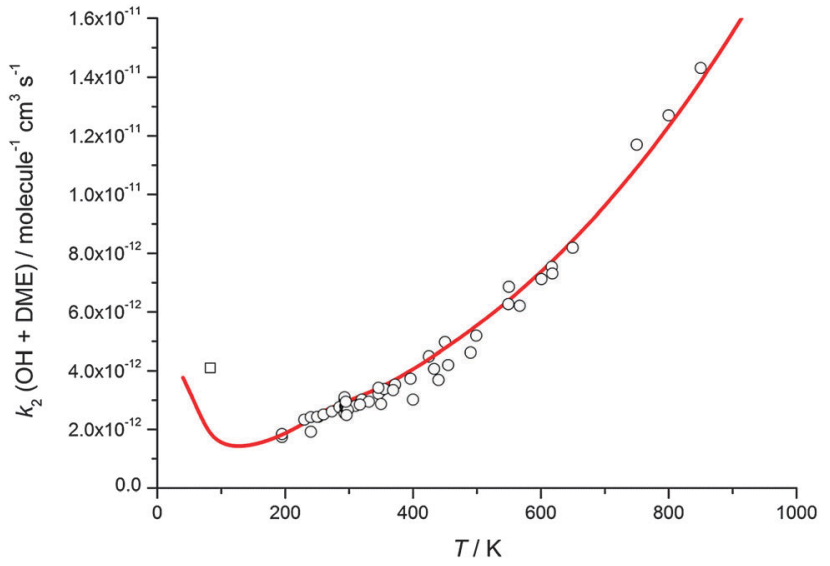

Fig. 9 MESMER master equation rate coefficients for $k_{2}(\mathrm{OH}+\mathrm{DME})$ versus temperature plotted alongside experimental data currently in the literature (references as for Fig. 3). The experimental data point (open square) is estimated from the $\mathrm{OH}$ recycling yield experiments in the presence of $\mathrm{O}_{2}$ at $93 \mathrm{~K}$, as described in the text.

kinetics of this reaction under the conditions of that study. The MESMER calculations were able to model the experimental rate coefficients within $16 \%$ between $200-1400 \mathrm{~K}$ with a model using a single transition state. ${ }^{32}$ The optimised geometric and energetic parameters have been used in this study to extend the MESMER calculations to the lower temperature experimental conditions of the current work, with the exception of the well depth for IM1 (OH-DME), which was not constrained by the higher temperature fits. In order to best fit the low temperature data it was found that the binding energy needed to be increased to $29.8 \mathrm{~kJ} \mathrm{~mol}^{-1}$. From Fig. 5 and 9 it can be seen that MESMER is able to reproduce the temperature behaviour over a wide range of temperatures, and also predicts the pressure dependence well for $T \sim 80 \mathrm{~K}$. Once again, the MESMER calculations predict that at zero-pressure there is a significant intercept corresponding to a pressure independent channel for the reaction of $\mathrm{OH}$ with DME, consistent with the experimental data demonstrating a yield of $\geq 50 \%$ for the $\mathrm{CH}_{3} \mathrm{COCH}_{2}$ abstraction product at this temperature.

\section{Discussion}

The rate coefficients below $150 \mathrm{~K}$ for both the reactions of $\mathrm{OH}$ with acetone and DME are significantly larger than observed previously at higher temperatures, and a pressure dependence provides evidence for an association channel with stabilisation into a weakly bound complex. At temperatures above $200 \mathrm{~K}$ the rate coefficients for both reactions increase with temperature and are pressure independent, consistent with reaction proceeding over an energy barrier. For the reaction of $\mathrm{OH}$ with DME, the rate coefficient shows significant curvature when plotted in Arrhenius form, and for the reaction of $\mathrm{OH}$ with acetone between 300 and $200 \mathrm{~K}$ the rate coefficients begin to become independent of temperature. In a previous study of the reaction of $\mathrm{OH}$ with methanol, ${ }^{5}$ a significant enhancement in the rate coefficient was also observed at very low temperatures. 
However, the reaction was found to be pressure independent, and observation of the $\mathrm{CH}_{3} \mathrm{O}$ radical reaction product demonstrated the role of tunnelling from the hydrogen-bonded complex through the activation barrier, supported by theoretical calculations.

The two transition state model developed by Greenwald et al., ${ }^{18,19}$ illustrated by the potential energy surface shown in Fig. 1, is useful to explore the role of the hydrogen-bonded prereactive complex at low temperatures and rationalise the enhancement in the rate constants at low temperatures. Using this two transition state model, simple expressions describing the high and low temperature limiting behaviour of system can be obtained. In the limit of low temperature, there is insufficient collision energy to access TS2 and so the transition state is located at TS1. The rate of dissociation of the complex becomes negligible compared to its rate of formation and the complex exhibits a significant lifetime and its concentration may become significant. Under these conditions the dominant bottleneck to reaction is TS1, and the observed rate coefficient is associated with the rate of formation of the complex IM1, which is limited by the capture rate coefficient for $\mathrm{OH}$ with the oxygenated VOC. The increased lifetime of IM1 will also enable stabilisation by collisions with the bath gas and facilitates product formation via tunnelling for these systems involving hydrogen atoms. Conversely in the limit of high temperature the complex rapidly dissociates back to reactants, and there is rapid equilibration between the reactants and the complex, and the rate determining step is crossing the barrier at TS2. The observed rate coefficient then reduces to the equilibrium constant for the formation of the complex multiplied by the rate coefficient for the complex crossing the barrier to reaction. The large increase in the observed rate constant below $200 \mathrm{~K}$ can be understood in terms of the controlling transitionstate moving to from TS2 to TS1. ${ }^{1}$

Unlike the $\mathrm{OH}+$ methanol reaction for which no pressure dependence was observed at very low temperatures, ${ }^{5}$ the rate determining step in the reaction of $\mathrm{OH}$ with acetone and DME reactions under similar conditions is the formation of the stabilized pre-reactive complex, IM1, evidenced by observation of a pressure dependence. The MESMER modelling shows that despite the weak binding energies of these complexes $\left(\sim 20 \mathrm{~kJ} \mathrm{~mol}^{-1}\right)$, and the relatively low total densities of the $\mathrm{N}_{2}$ bath gas used $\left(5-20 \times 10^{16}\right.$ molecule $\left.\mathrm{cm}^{-3}\right)$, collisional stabilization of the energized complex occurs readily on the timescale on the experimental timescale (up to $300 \mu$ s reaction time in the Laval expansion). Inspection of Fig. 4 and 5 shows that for a given pressure and temperature, the rate coefficients for the reaction of $\mathrm{OH}$ with acetone are closer to the high pressure limit than for DME. This behaviour can be readily understood as acetone is a larger molecule and hence the number of vibrational modes, and hence the density of states of the complex will be larger relative to that of the separated reagents, and so the lifetime of the complex will be longer. As was shown in Section 4.2 above, the best-fit parameters from comparison of the MESMER model with the experimental data included a well-depth for $\mathrm{OH}$-acetone which was considerably deeper than calculated from ab initio methods, as a result of the methodology used in calculating a density of states which was too low. With increasingly sophisticated treatment of hindered rotational modes and vibrational anharmonicity, ${ }^{5}$ the calculated density of states of the complex would be expected to increase, resulting in an increase in the predicted rate coefficients using MESMER. Hence the well depth obtained from the best fit of the MESMER model to the experimental data would become closer to those values calculated with ab initio methods.

Although it is established that tunnelling is not the rate determining step in the reactions of $\mathrm{OH}$ with either acetone or DME, there is evidence from the non-zero intercepts of Fig. 4 and 5 at zero pressure of a bimolecular channel for both reactions that is pressure independent and due to tunnelling. As shown in Fig. 7, the MESMER calculations show that tunnelling is required in order to model the system successfully over the wide range of temperatures for which kinetic data are available. For the reaction of $\mathrm{OH}$ with $\mathrm{DME}$ there is additional evidence of the formation of the $\mathrm{CH}_{3} \mathrm{OCH}_{2}$ product, and hence for efficient quantum mechanical tunnelling.

In addition, the master equation can calculate the time dependence of any of the species, and in Fig. 10 these are shown for the $\mathrm{OH}$ reactant, the pre-reaction complex, IM1, and the post-reaction complex IM2 (the step from IM2 to bimolecular products was not considered in the simulations). The lifetime of the pre-reactive complex is considerable, peaking at around $100 \mu$ s. Once the stabilised complex is formed, on longer timescales it will eventually all be converted to products via quantum mechanical tunnelling, but does not have sufficient energy to convert back to the reagents. There is always competition at low temperatures between stabilization of IM1 and tunnelling through the barrier, and depending upon the density of states in IM1, the kinetic behaviour for reactions of $\mathrm{OH}$ with OVOCs will be at different points on the fall-off curve for a given combination of pressure and temperature. For the smaller methanol molecule studied previously the master equation calculated a rate coefficient for the loss of $\mathrm{OH}$ due to stabilisation

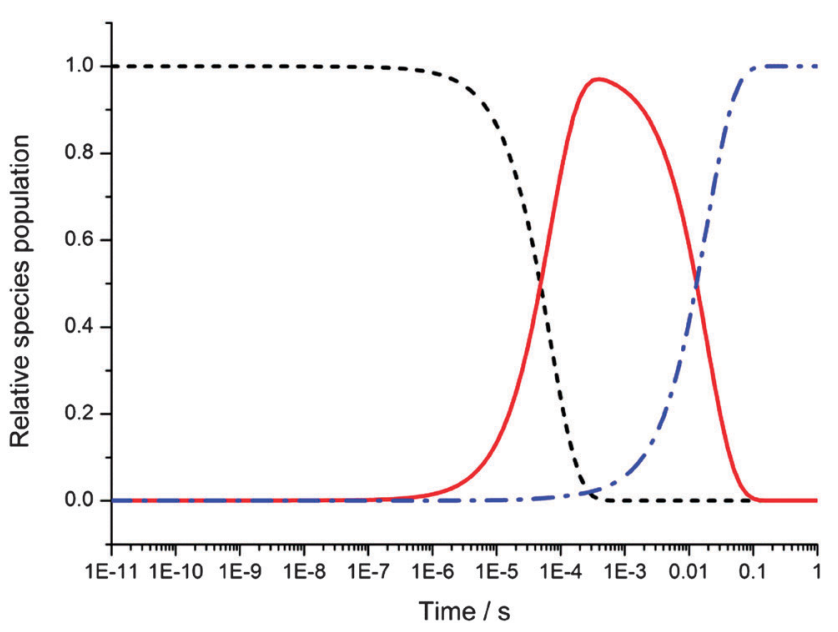

Fig. 10 Time evolution of the concentrations of $\mathrm{OH}$ (black dashed line), IM1 (red full line) and IM2 (blue dot/dash line) for the reaction of $\mathrm{OH}$ with acetone calculated by MESMER at $80 \mathrm{~K}$ and a total gas density of $\left[\mathrm{N}_{2}\right]=$ $6 \times 10^{16}$ molecule $\mathrm{cm}^{-3}$. See text for details. 
of the complex to be $\sim 1 \times 10^{-13}$ molecule ${ }^{-1} \mathrm{~cm}^{3} \mathrm{~s}^{-1}$ at $80 \mathrm{~K}$ and a similar total density, and the rate coefficient was dominated by tunnelling. ${ }^{5}$ However, as seen in Fig. 4 and 5 for the $\mathrm{OH}$ reactions with acetone and DME under similar conditions, the reaction rate coefficients are in the fall-off region with a dominant contribution to the rate coefficient from collisional stabilisation.

\section{Conclusions}

The rate coefficients for the reactions of $\mathrm{OH}$ with acetone and dimethyl ether (DME) were studied between 63-148 K using a pulsed Laval nozzle apparatus. For both reactions, a marked inverse temperature dependence was observed, with rate coefficients considerably higher than those obtained previously at $\sim 200 \mathrm{~K}$. For both reactions the rate coefficient was found to be pressure dependent at the very low temperatures used, in contrast to our previous study of the reaction of $\mathrm{OH}$ with methanol for which the rate coefficient was independent of pressure, and for which a tunnelling mechanism was shown to dominate. The pressure dependence suggests a LindemannHinshelwood type mechanism operates for these reactions, with collisional stabilisation of the $\mathrm{OH}-\mathrm{OVOC}$ pre-reaction complex resulting in the observed loss of $\mathrm{OH}$ radicals. For acetone, the rate coefficient at $93 \mathrm{~K}$ did not show a kinetic isotope effect, showing that tunnelling is not dominating the mechanism. However, for both reactions extrapolation of the pressure dependent rate coefficients to zero pressure shows an intercept which provides evidence for a contribution from a pressure independent bimolecular channel leading to abstraction products via tunnelling. In the case of the reaction of $\mathrm{OH}$ with DME, further evidence for tunnelling to form the abstraction product $\mathrm{CH}_{3} \mathrm{OCH}_{2}$ was obtained via measurement of a smaller rate coefficient in the presence of $\mathrm{O}_{2}$ due to recycling of $\mathrm{OH}$ from the reaction of $\mathrm{CH}_{3} \mathrm{OCH}_{2}$ with $\mathrm{O}_{2}$, and a lower limit for the yield of $\mathrm{CH}_{3} \mathrm{OCH}_{2}$ of $\sim 0.5$ was obtained. The experimental measurements were supported by master equation calculations using the MESMER code which included a treatment of quantum mechanical tunnelling, and using optimised energies for the stationary points on the minimum energy path for reaction obtained by fitting to the available data over a wide range of temperatures. MESMER was able to reproduce the large increase in the rate coefficients at very low temperatures for both reactions as well as fitting the data at higher temperatures, and was able to model the pressure dependence well at very low temperatures. The calculations support the mechanism for these reactions of the formation of a pre-reactive complex (IM1) for which the dominant fate is collisional stabilisation, but with a contribution from tunnelling to form abstraction products. MESMER was also used to calculate the rate coefficients at $1 \times 10^{6}$ molecule $^{-1}$ and at temperatures down to $10 \mathrm{~K}$, typical of the molecular clouds or the interstellar medium, and showed that the rate coefficient for tunnelling was $\sim 10 \%$ of the collision rate. Therefore, similar to the reaction of $\mathrm{OH}$ with methanol for which tunnelling was dominant under the conditions in space, the reactions of $\mathrm{OH}$ with acetone and DME are also likely to be fast under these conditions, and may be important in such an environment. The reactions of $\mathrm{OH}$ with methanol, acetone, DME and also methyl ethyl ketone (MEK) all show similar behaviour, and we hypothesise that reactions of $\mathrm{OH}$ with oxygenated VOCs, for which a hydrogen-bonded pre-reactive complex is formed, will behave similarly at very low temperatures, and demonstrate a contribution from tunnelling.

\section{Acknowledgements}

R.J.S. and R.L.C. acknowledge the Natural Environment Research Council for funding of PhD studentships. We would also like to thank Dr A. Goddard for technical assistance.

\section{References}

1 R. J. Shannon, S. Taylor, A. Goddard, M. A. Blitz and D. E. Heard, Phys. Chem. Chem. Phys., 2010, 12, 13511-13514.

2 J. Bouwman, M. Fournier, I. R. Sims, S. R. Leone and K. R. Wilson, J. Phys. Chem. A, 2013, 117, 5093-5105.

3 H. Sabbah, L. Biennier, I. R. Sims, Y. Georgievskii, S. J. Klippenstein and I. W. M. Smith, Science, 2007, 317, 102-105.

4 E. Vöhringer-Martinez, B. Hansmann, H. Hernandez, J. S. Francisco, J. Troe and B. Abel, Science, 2007, 315, 497-501.

5 R. J. Shannon, M. A. Blitz, A. Goddard and D. E. Heard, Nat. Chem., 2013, 5, 745-749.

6 J. Daranlot, M. Jorfi, C. Xie, A. Bergeat, M. Costes, P. Caubet, D. Xie, H. Guo, P. Honvault and K. M. Hickson, Science, 2011, 334, 1538-1541.

7 B. R. Rowe, G. Dupeyrat, J. B. Marquette and P. Gaucherel, J. Chem. Phys., 1984, 80, 4915-4921.

8 I. W. M. Smith and B. R. Rowe, Acc. Chem. Res., 2000, 33, 261-268.

9 I. W. M. Smith, Angew. Chem., Int. Ed., 2006, 45, 2842-2861.

10 I. W. M. Smith, E. Herbst and Q. Chang, Mon. Not. R. Astron. Soc., 2004, 350, 323-330.

11 I. W. Smith, A. M. Sage, N. M. Donahue, E. Herbst and D. Quan, Faraday Discuss., 2006, 133, 137-156.

12 A. M. Rouhi, Amoco, Haldor Topsoe Develop Dimethyl Ether As Alternative Diesel Fuel, Chem. Eng. News, 1995, 73, 37-39.

13 I. W. M. Smith, A. M. Sage, N. M. Donahue, E. Herbst and D. Quan, Faraday Discuss., 2006, 133, 137-156.

14 F. Combes, M. Gerin, A. Wootten, G. Wlodarczak, F. Clausset and P. J. Encrenaz, Astron. Astrophys., 1987, 180, L13-L16.

15 D. L. Thacker, W. J. Wilson and A. H. Barrett, Astrophys. J., 1970, 161, L191-L197.

16 G. Winnewisser and F. F. Gardner, Astron. Astrophys., 1976, 48, 159-161.

17 V. Wakelam, E. Herbst, J.-C. Loison, I. Smith, V. Chandrasekaran, B. Pavone, N. Adams, M.-C. Bacchus-Montabonel, A. Bergeat and K. Béroff, Astrophys. J., Suppl. Ser., 2012, 199, 21.

18 E. E. Greenwald, S. W. North, Y. Georgievskii and S. J. Klippenstein, J. Phys. Chem. A, 2007, 111, 5582-5592. 
19 E. E. Greenwald, S. W. North, Y. Georgievskii and S. J. Klippenstein, J. Phys. Chem. A, 2005, 109, 6031-6044.

20 F. Caralp, W. Forst, E. Henon, A. Bergeat and F. Bohr, Phys. Chem. Chem. Phys., 2006, 8, 1072-1078.

21 T. J. Wallington and M. J. Kurylo, J. Phys. Chem., 1987, 91, 5050-5054.

22 I. W. M. Smith and A. R. Ravishankara, J. Phys. Chem. A, 2002, 106, 4798-4807.

23 R. J. Shannon, S. Taylor, A. Goddard, M. A. Blitz and D. E. Heard, Phys. Chem. Chem. Phys., 2010, 12, 13511-13514.

24 D. R. Glowacki, C.-H. Liang, C. Morley, M. J. Pilling and S. H. Robertson, J. Phys. Chem. A, 2012, 116, 9545-9560.

25 S. E. Taylor, A. Goddard, M. A. Blitz, P. A. Cleary and D. E. Heard, Phys. Chem. Chem. Phys., 2008, 10, 422-437.

26 S. A. Carr, M. T. Baeza-Romero, M. A. Blitz, B. J. S. Price and P. W. Seakins, Int. J. Chem. Kinet., 2008, 40, 504-514.

27 J. T. Bartis and B. Widom, J. Chem. Phys., 1974, 60, 3474-3482.

28 M. A. Blitz, K. J. Hughes, M. J. Pilling and S. H. Robertson, J. Phys. Chem. A, 2006, 110, 2996-3009.

29 J. A. Miller, S. J. Klippenstein, S. H. Robertson, M. J. Pilling and N. J. B. Green, Phys. Chem. Chem. Phys., 2009, 11, 1128-1137.

30 S. Robertson, M. Pilling, D. Baulch and N. Green, J. Phys. Chem., 1995, 99, 13452-13460.

31 E. Henon, S. Canneaux, F. Bohr and S. Dobe, Phys. Chem. Chem. Phys., 2003, 5, 333-341.

32 S. A. Carr, T. J. Still, M. Blitz, A. J. Eskola, M. J. Pilling, P. Seakins, R. Shannon, B. Wang and S. H. Robertson, J. Phys. Chem. A, 2013, 117, 11142-11154.

33 M. J. Frisch, G. W. Trucks, H. B. Schlegel, G. E. Scuseria, M. A. Robb, J. R. Cheeseman, J. A. Montgomery Jr, T. Vreven, K. N. Kudin, J. C. Burant, J. M. Millam, S. S. Iyengar, J. Tomasi, V. Barone, B. Mennucci, M. Cossi, G. Scalmani, N. Rega, G. A. Petersson, H. Nakatsuji, M. Hada, M. Ehara, K. Toyota, R. Fukuda, J. Hasegawa, M. Ishida, T. Nakajima, Y. Honda, O. Kitao, H. Nakai, M. Klene, X. Li, J. E. Knox, H. P. Hratchian, J. B. Cross, V. Bakken, C. Adamo, J. Jaramillo, R. Gomperts, R. E. Stratmann, O. Yazyev, A. J. Austin, R. Cammi, C. Pomelli, J. W. Ochterski, P. Y. Ayala, K. Morokuma, G. A. Voth, P. Salvador, J. J. Dannenberg, V. G. Zakrzewski, S. Dapprich, A. D. Daniels, M. C. Strain, O. Farkas, D. K. Malick, A. D. Rabuck, K. Raghavachari, J. B. Foresman, J. V. Ortiz, Q. Cui, A. G. Baboul, S. Clifford, J. Cioslowski, B. B. Stefanov, G. Liu, A. Liashenko, P. Piskorz, I. Komaromi, R. L. Martin, D. J. Fox, T. Keith, M. A. Al-Laham, C. Y. Peng, A. Nanayakkara, M. Challacombe, P. M. W. Gill, B. Johnson, W. Chen, M. W. Wong, C. Gonzalez and J. A. Pople, Gaussian 03, Revision D.02, Gaussian, Inc., Wallingford, CT, 2004.

34 S. Sharma, S. Raman and W. H. Green, J. Phys. Chem. A, 2010, 114, 5689-5701.

35 W. H. Miller, J. Am. Chem. Soc., 1979, 101, 6810-6814.

36 B. C. Garrett and D. G. Truhlar, J. Phys. Chem., 1979, 83, 2921-2926.

37 Y. Georgievskii and S. J. Klippenstein, J. Chem. Phys., 2005, 122, 194103.

38 M. J. Pilling and S. H. Robertson, Annu. Rev. Phys. Chem., 2003, 54, 245-275.

39 T. Yamada, P. H. Taylor, A. Goumri and P. Marshall, J. Chem. Phys., 2003, 119, 10600-10606.

40 S. Vandenberk, L. Vereecken and J. Peeters, Phys. Chem. Chem. Phys., 2002, 4, 461-466.

41 A. J. Eskola, S. A. Carr, M. A. Blitz, M. J. Pilling and P. W. Seakins, Chem. Phys. Lett., 2010, 487, 45-50.

42 J. Lockhart, M. A. Blitz, D. E. Heard, P. W. Seakins and R. J. Shannon, J. Phys. Chem. A, 2013, 117, 5407-5418.

43 D. R. Glowacki, J. Lockhart, M. A. Blitz, S. J. Klippenstein, M. J. Pilling, S. H. Robertson and P. W. Seakins, Science, 2012, 337, 1066-1069.

44 M. T. B. Romero, M. A. Blitz, D. E. Heard, M. J. Pilling, B. Price and P. W. Seakins, Chem. Phys. Lett., 2005, 408, 232-236.

45 T. Gierczak, M. K. Gilles, S. Bauerle and A. R. Ravishankara, J. Phys. Chem. A, 2003, 107, 5014-5020.

46 E. F. van Dishoeck, G. A. Blake, B. T. Draine and J. I. Lunine, The chemical evolution of protostellar and protoplanetary matter, in Protostars and Planets III, ed. E. H. Levy and J. I. Lunine, University of Arizona Press, 1993, pp. 163-241.

47 A. Mellouki, S. Teton and G. Lebras, Int. J. Chem. Kinet., 1995, 27, 791-805.

48 M. Arif, B. Dellinger and P. H. Taylor, J. Phys. Chem. A, 1997, 101, 2436-2441.

49 A. Bonard, V. Daele, J. L. Delfau and C. Vovelle, J. Phys. Chem. A, 2002, 106, 4384-4389.

50 R. D. Cook, D. F. Davidson and R. K. Hanson, J. Phys. Chem. A, 2009, 113, 9974-9980.

51 T. J. Wallington, P. Dagaut, R. Z. Liu and M. J. Kurylo, Int. J. Chem. Kinet., 1988, 20, 541-547.

52 M. Wollenhaupt, S. A. Carl, A. Horowitz and J. N. Crowley, J. Phys. Chem. A, 2000, 104, 2695-2705.

53 T. Gierczak, M. K. Gilles, S. Bauerle and A. R. Ravishankara, J. Phys. Chem. A, 2003, 107, 5014-5020.

54 T. J. Wallington, R. Z. Liu, P. Dagaut and M. J. Kurylo, Int. J. Chem. Kinet., 1988, 20, 41-49.

55 J. Sehested, T. E. Møgelberg, T. J. Wallington, E. W. Kaiser and O. J. Nielsen, J. Phys. Chem., 1996, 100, 17218-17225. 